\title{
Linkage disequilibrium mapping for grain Fe and Zn enhancing QTLs useful for nutrient dense rice breeding
}

\author{
S. K. Pradhan ${ }^{1 *+}$, E. Pandit ${ }^{1 *+}$, S. Pawar ${ }^{1}$, R. Naveenkumar' ${ }^{1}$ S. R. Barik' , S. P. Mohanty ${ }^{1}$, D. K. Nayak', S. K. Ghritlahre ${ }^{1}$, \\ D. Sanjiba Rao ${ }^{2}$, J. N. Reddy ${ }^{1}$ and S. S. C. Patnaik
}

\begin{abstract}
Background: High yielding rice varieties are usually low in grain iron ( $\mathrm{Fe}$ ) and zinc ( $\mathrm{Zn}$ ) content. These two micronutrients are involved in many enzymatic activities, lack of which cause many disorders in human body. Biofortification is a cheaper and easier way to improve the content of these nutrients in rice grain.

Results: A population panel was prepared representing all the phenotypic classes for grain Fe-Zn content from 485 germplasm lines. The panel was studied for genetic diversity, population structure and association mapping of grain Fe-Zn content in the milled rice. The population showed linkage disequilibrium showing deviation of HardyWeinberg's expectation for Fe-Zn content in rice. Population structure at $\mathrm{K}=3$ categorized the panel population into distinct sub-populations corroborating with their grain Fe-Zn content. STRUCTURE analysis revealed a common primary ancestor for each sub-population. Novel quantitative trait loci (QTLs) namely qFe3.3 and qFe7.3 for grain Fe and qZn2.2, qZn8.3 and qZn12.3 for Zn content were detected using association mapping. Four QTLs, namely qFe3.3, $q F e 7.3, q F e 8.1$ and $q F e 12.2$ for grain Fe content were detected to be co-localized with qZn3.1, qZn7, qZn8.3 and qZn12.3 QTLs controlling grain Zn content, respectively. Additionally, some Fe-Zn controlling QTLs were co-localized with the yield component QTLs, qTBGW, OSSPL14 and qPN. The QTLs qFe1.1, qFe3.1, qFe5.1, qFe7.1, qFe8.1, qZn6, qZn7 and gRMm9-1 for grain Fe-Zn content reported in earlier studies were validated in this study.

Conclusion: Novel QTLs, qFe3.3 and qFe7.3 for grain Fe and qZn2.2, qZn8.3 and qZn12.3 for Zn content were detected for these two traits. Four Fe-Zn controlling QTLs and few yield component QTLs were detected to be colocalized. The QTLs, qFe1.1, qFe3.1, qFe5.1, qFe7.1, qFe8.1, qFe3.3, qFe7.3, qZn6, qZn7, qZn2.2, qZn8.3 and qZn12.3 will be useful for biofortification of the micronutrients. Simultaneous enhancement of Fe-Zn content may be possible with yield component traits in rice.
\end{abstract}

Keywords: Association study, Linkage disequilibrium, Grain Fe content, Grain Zn content, Biofortification

\section{Background}

Majority of the global population consume rice daily, particularly in Asiatic countries. But, rice grain is poor source of micronutrients such as iron $(\mathrm{Fe})$ and zinc $(\mathrm{Zn})$. The practice of consuming polished rice as a staple food in India aggravates malnutrition. Substantial amounts of iron and zinc are removed during milling. The polished rice contains around $2 \mathrm{mg} \mathrm{kg}^{-1} \mathrm{Fe}$ while the recommended

\footnotetext{
*Correspondence: pradhancrri@gmail.com; elsambio@gmail.com

${ }^{+}$S. K. Pradhan and E. Pandit contributed equally to this work.

${ }^{1}$ ICAR-National Rice Research Institute, Cuttack, Odisha, India

Full list of author information is available at the end of the article
}

dietary intake is $10-15 \mathrm{mg} \mathrm{kg}^{-1}$. Similarly, polished rice contains around $12 \mathrm{mg} \mathrm{kg}^{-1}$ of $\mathrm{Zn}$, whereas the recommended intake for humans is $12-15 \mathrm{mg} \mathrm{kg}^{-1}$ [1]. Iron is an important constituent of haemoglobin in red blood cells and is essential for the proper functioning of several enzymes in the body. Zinc is required for the metabolic activity of 300 enzymes, and is essential for enzymes involved in cell division, protein synthesis and growth [2]. To date, proper attention has not been given for improvement of these micronutrients in rice grain. Literature survey on existence of grain $\mathrm{Fe}$ and $\mathrm{Zn}$ content diversity reported in rice is high in natural rice germplasms [3-13].

(c) The Author(s). 2020 Open Access This article is distributed under the terms of the Creative Commons Attribution 4.0 International License (http://creativecommons.org/licenses/by/4.0/), which permits unrestricted use, distribution, and reproduction in any medium, provided you give appropriate credit to the original author(s) and the source, provide a link to the Creative Commons license, and indicate if changes were made. The Creative Commons Public Domain Dedication waiver (http://creativecommons.org/publicdomain/zero/1.0/) applies to the data made available in this article, unless otherwise stated. 
Increasing the iron and zinc content in rice grain through breeding is cheaper and an easier option to reduce malnutrition in the developing countries. Increasing Fe and $\mathrm{Zn}$ content in rice is possible by utilizing elite germplasms possessing enormous genetic potential for grain $\mathrm{Fe}-\mathrm{Zn}$ content in rice breeding program $[5,6,13-15]$.

Popular rice varieties usually contain lesser micronutrients in grains compared to the traditional cultivars and landraces [16, 17]. QTLs responsible for further enhancing these nutrients in the high yielding rice varieties need to be identified and utilized. Through classical breeding approach, a very limited success could be achieved in this context. Earlier reports on low heritability of micronutritients concentration, negative relationship with high grain yield and genotype and environment interaction were the main limitations of genetic enhancement for these nutrients [18-21]. However, the development of CR Dhan 311, DRR Dhan 45 and Chhattishgarh Zinc Rice-1 are the recent examples of high yielding varieties in India with high grain $\mathrm{Zn}$ content. But, these achievements were through non-targeted classical breeding approach. Success in breeding for micronutrients enrichment is limited due to involvement of many quantitative trait loci (QTLs) with small effects and interacts highly with the environmental factors. Precision breeding is a better approach for enriching the elite rice varieties with desired micronutrients. The availability of information on robust markers for different QTLs and potential donors are pre-requisite for the success of precision breeding. Several QTLs for grain Fe and $\mathrm{Zn}$ related traits have been reported in rice from different genetic backgrounds of intraspecific and interspecific crosses $[6,9,13-15,18-36]$ are hardly being in use in modern molecular breeding for $\mathrm{Fe}-\mathrm{Zn}$ enhancement.

Mapping reports of Fe- $\mathrm{Zn}$ content in rice indicated the use of various bi-parental mapping populations for detecting the genes controlling these two micronutrients. Gene mapping using bi-parental mapping populations is more time consuming, costlier and lesser resolution than association mapping [17, 37-40]. These limitations can be overcome by linkage disequilibrium (LD) mapping or association mapping. Association mapping is based on linkage disequilibrium or variations existing in natural or developed populations $[17,37,39,40]$. The main purpose of association mapping here is to estimate the correlations between molecular markers with the grain $\mathrm{Fe}$ and $\mathrm{Zn}$ content in a panel containing representative rice population that possess considerable amount of variability for these two traits. Only few research reports on association mapping for grain Fe-Zn content are available [41, 42]. But, report on QTL mapping through association mapping in rice are available for grain yield and agronomic traits [29, $41,43,44]$; seedling stage cold tolerance [40, 45]; cold tolerance at germination and booting stages [46]; high temperature stress tolerance [39]; grain quality traits [47]; salinity tolerance [48]; drought tolerance traits [49, 50]; early seedling vigour [51] and grain protein content [17]. However, the information on association mapping for grain Fe and $\mathrm{Zn}$ content in rice is scarce. In this study, a panel population containing 102 genotypes shortlisted from 485 germplasm lines were analyzed using 100 SSR and gene specific molecular markers for genetic diversity, population structure and association mapping for grain $\mathrm{Fe}$ and $\mathrm{Zn}$ content.

\section{Results}

Phenotyping of the panel population for grain Fe and $\mathrm{Zn}$ content in milled rice

The panel population was prepared by shortlisting genotypes from different phenotypic groups based on screening results of 485 germplasm lines for $\mathrm{Fe}-\mathrm{Zn}$ content. Almost equal proportions of genotypes were pooled from each $\mathrm{Fe}-\mathrm{Zn}$ phenotypic group along with the check varieties for constitution of the panel containing 102 germplasm lines. The mean iron content in of the panel population varied from 1.07 to 5.38 $\mathrm{mg} \mathrm{kg}^{-1}$ in the milled rice phenotyping results of wet seasons, 2016 and 2017. A higher Fe-content of $>4$ $\mathrm{mg} \mathrm{kg}^{-1}$ in milled rice was observed in 13 genotypes viz., Chittimuthylu, IET25450, IET 24775, IET 24760, IET 24316, BPT5204, IET 23832, Kalanamak, IET25441 and IET25465, IET 23829 and IET 24779 (Additional file 3: Table S1). The check varieties for micronutrients viz., Chittimuthylu and Kalanamak showed mean grain Fe content of 4.8 and $4.48 \mathrm{mg} \mathrm{kg}^{-}$ ${ }^{1}$, respectively. Genotypes containing $\geq 25 \mathrm{mg} \mathrm{kg}^{-1}$ zinc and /or $\geq 10 \mathrm{mg} \mathrm{kg}^{-1}$ iron in brown rice along with at par or higher grain yield compare to the yield checks are considered desirable biofortified rice lines. While comparing in milled rice, genotype containing $\geq 4 \mathrm{mg}$ $\mathrm{kg}^{-1} \mathrm{Fe}$ and $\geq 20 \mathrm{mg} \mathrm{kg}^{-1} \mathrm{Zn}$ and producing higher yield than yield check variety is considered as desirable genotypes. It is observed that the genotypes enlisted in the Table containing $>4 \mathrm{mg} \mathrm{kg}^{-1} \mathrm{Fe}$ in milled rice were found to be with $>12 \mathrm{mg} \mathrm{kg}^{-1}$ in brown rice. Similarly, genotypes present in the panel with $>20 \mathrm{mg} \mathrm{kg}^{-1} \mathrm{Zn}$ content in milled rice showed $>25 \mathrm{mg} \mathrm{kg}^{-1}$ in brown rice. From the results, nine biofortified rice genotypes, two micronutrients check cultivars and landrace Sadakadam showed more than $4 \mathrm{mg} \mathrm{kg}^{-1} \mathrm{Fe}$ in milled rice and categorized under high Fe containing genotypes in our study (Table 1). Forty two genotypes comprised of the moderate group (3-4 $\mathrm{mg} \mathrm{kg}^{-1} \mathrm{Fe}$ in milled rice) while 48 showed low values $\left(<3 \mathrm{mg} \mathrm{kg}^{-1} \mathrm{Fe}\right)$. Mean zinc content in milled rice varied from $7.43-27.97 \mathrm{mg} \mathrm{kg}^{-1}$ showing $\geq 20 \mathrm{mg} \mathrm{kg}^{-1}$ in fourteen genotypes (Additional file 3: Table S1). These fourteen genotypes were categorized under high grain $\mathrm{Zn}$ containing rice. 
Table 1 Mean estimate values of days to 50\% flowering, grain Fe- Zn content, panicles/m² and grain yield of 102 genotypes including biofortified lines and check varieties studied during wet season, 2016 \& 2017

\begin{tabular}{|c|c|c|c|c|c|c|}
\hline SI. No. & National testing No./Accession No./Name of the genotype & $\begin{array}{l}\text { Days to } 50 \% \\
\text { flowering }\end{array}$ & $\begin{array}{l}\text { Iron content } \\
\text { in ppm }\end{array}$ & $\begin{array}{l}\text { Zinc content } \\
\text { in ppm }\end{array}$ & Panicles $/ \mathrm{m}^{2}$ & $\begin{array}{l}\text { Grain } \\
\text { yield } \\
\text { (kg/ha) }\end{array}$ \\
\hline 1 & IET23829 & 99 & 4.18 & 18.73 & 282 & 3950 \\
\hline 2 & IR64 & 91 & 3.97 & 17.24 & 281 & 4250 \\
\hline 3 & IET23834 & 88 & 3.5 & 18.8 & 264 & 3450 \\
\hline 4 & Kalanamak & 106 & 4.48 & 25.91 & 265 & 2962 \\
\hline 5 & IET23824 & 90 & 3.75 & 20.1 & 262 & 3771 \\
\hline 6 & IET23832 & 97 & 4.15 & 18.43 & 266 & 4068 \\
\hline 7 & Chittimuthyalu & 110 & 4.8 & 22.03 & 283 & 3620 \\
\hline 8 & IET24780 & 94 & 3.21 & 14.09 & 295 & 4552 \\
\hline 9 & BPT5204 & 111 & 4.11 & 15.46 & 285 & 4431 \\
\hline 10 & IET24771 & 107 & 3.9 & 15.68 & 286 & 4958 \\
\hline 11 & IET24766 & 94 & 3.41 & 15.2 & 279 & 4281 \\
\hline 12 & IET24316 & 87 & 4.04 & 18.25 & 264 & 3532 \\
\hline 13 & IET24391 & 110 & 2.99 & 20.11 & 249 & 3984 \\
\hline 14 & IET24777 & 96 & 2.73 & 14.8 & 295 & 4848 \\
\hline 15 & IET24760 & 110 & 4.11 & 18.88 & 303 & 4693 \\
\hline 16 & IET24775 & 110 & 4.33 & 17.47 & 284 & 4623 \\
\hline 17 & IET24783 & 96 & 3.35 & 17.18 & 275 & 4883 \\
\hline 18 & IET24544 & 98 & 2.49 & 16.4 & 286 & 4899 \\
\hline 19 & IET24336 & 93 & 2.77 & 17.37 & 269 & 4598 \\
\hline 20 & IET24772 & 96 & 3.26 & 17.8 & 287 & 4285 \\
\hline 21 & IET24557 & 107 & 3.97 & 18.34 & 288 & 4079 \\
\hline 22 & IET24779 & 102 & 4.02 & 19.67 & 280 & 4283 \\
\hline 23 & IET24774 & 104 & 2.93 & 17.4 & 293 & 4685 \\
\hline 24 & IET24787 & 97 & 3.62 & 17.2 & 280 & 3826 \\
\hline 25 & IET25441 & 101 & 4.06 & 16.48 & 275 & 4258 \\
\hline 26 & IET25443 & 109 & 3.6 & 19.73 & 284 & 3629 \\
\hline 27 & IET25444 & 111 & 3.45 & 16.31 & 257 & 4229 \\
\hline 28 & IET25445 & 106 & 3.57 & 14.55 & 287 & 4399 \\
\hline 29 & IET25446 & 94 & 3.95 & 21.02 & 284 & 3857 \\
\hline 30 & IET25447 & 105 & 3.5 & 15.57 & 290 & 4748 \\
\hline 31 & IET25449 & 98 & 3.19 & 15.2 & 266 & 4379 \\
\hline 32 & IET25450 & 99 & 4.41 & 23.43 & 282 & 3133 \\
\hline 33 & IET25452 & 106 & 3.41 & 16.42 & 280 & 4490 \\
\hline 34 & IET25453 & 93 & 3.03 & 15.95 & 269 & 4515 \\
\hline 35 & IET25454 & 87 & 3.33 & 19.27 & 227 & 2550 \\
\hline 36 & IET25457 & 99 & 2.9 & 16.71 & 246 & 3582 \\
\hline 37 & IET25459 & 102 & 2.97 & 15.22 & 272 & 4479 \\
\hline 38 & IET25460 & 105 & 3.41 & 14.22 & 284 & 5210 \\
\hline 39 & IET25461 & 95 & 3.53 & 20.14 & 256 & 3767 \\
\hline 40 & IET25463 & 98 & 3.09 & 15.32 & 276 & 4843 \\
\hline 41 & IET25464 & 94 & 2.76 & 16.2 & 243 & 3392 \\
\hline 42 & IET25465 & 101 & 4.19 & 20.36 & 265 & 3656 \\
\hline
\end{tabular}


Table 1 Mean estimate values of days to 50\% flowering, grain Fe- Zn content, panicles/m² and grain yield of 102 genotypes including biofortified lines and check varieties studied during wet season, 2016 \& 2017 (Continued)

\begin{tabular}{|c|c|c|c|c|c|c|}
\hline SI. No. & National testing No./Accession No./Name of the genotype & $\begin{array}{l}\text { Days to } 50 \% \\
\text { flowering }\end{array}$ & $\begin{array}{l}\text { Iron content } \\
\text { in ppm }\end{array}$ & $\begin{array}{l}\text { Zinc content } \\
\text { in ppm }\end{array}$ & Panicles $/ \mathrm{m}^{2}$ & $\begin{array}{l}\text { Grain } \\
\text { yield } \\
\text { (kg/ha) }\end{array}$ \\
\hline 43 & Gontra Bidhan3 & 106 & 2.7 & 11.1 & 251 & 4050 \\
\hline 44 & IET25469 & 95 & 3.15 & 18.57 & 276 & 4054 \\
\hline 45 & IET25470 & 96 & 2.98 & 18.1 & 263 & 4772 \\
\hline 46 & IET25471 & 103 & 3.44 & 16.87 & 265 & 4276 \\
\hline 47 & DRRH3 & 101 & 2.53 & 13.88 & 253 & 5162 \\
\hline 48 & IET25472 & 92 & 3.78 & 26.96 & 275 & 3059 \\
\hline 49 & IET25473 & 103 & 3.35 & 16.62 & 280 & 5002 \\
\hline 50 & IET25474 & 105 & 3.26 & 18.95 & 269 & 4125 \\
\hline 51 & IET25475 & 91 & 3.37 & 23.35 & 267 & 3881 \\
\hline 52 & IET25477 & 101 & 3.13 & 24.28 & 284 & 3620 \\
\hline 53 & IET25478 & 105 & 2.94 & 16 & 271 & 4209 \\
\hline 54 & IET25479 & 106 & 3.94 & 16.84 & 290 & 4424 \\
\hline 55 & Lalmeeta & 118 & 2.79 & 12.66 & 252 & 3430 \\
\hline 56 & Abhimanyu & 119 & 3.78 & 10.44 & 272 & 3810 \\
\hline 57 & Kalobhutia & 121 & 3.96 & 11.7 & 246 & 3360 \\
\hline 58 & Sadakajam & 115 & 4.17 & 16.32 & 246 & 3210 \\
\hline 59 & Geetanjali & 128 & 3.18 & 11.94 & 280 & 3950 \\
\hline 60 & Kakhru & 127 & 2.85 & 13.56 & 254 & 3740 \\
\hline 61 & Boanti & 120 & 3.18 & 13.56 & 248 & 3430 \\
\hline 62 & Tulsimukul & 120 & 3.99 & 14.1 & 226 & 2830 \\
\hline 63 & Kokilpatri & 112 & 2.94 & 11.52 & 252 & 3280 \\
\hline 64 & Basmatikarnal & 125 & 3.21 & 12.3 & 242 & 3120 \\
\hline 65 & Kalonunia & 132 & 3.84 & 13.2 & 218 & 2930 \\
\hline 66 & SafedLuchai2 & 108 & 3.69 & 16.5 & 220 & 2940 \\
\hline 67 & Bankra & 137 & 2.49 & 12.24 & 248 & 3320 \\
\hline 68 & Moongi & 126 & 2.85 & 11.7 & 198 & 2830 \\
\hline 69 & Swarnakranti & 124 & 3.42 & 10.86 & 274 & 3810 \\
\hline 70 & Kalojeera & 99 & 3.69 & 9.96 & 188 & 2820 \\
\hline 71 & Ketekijoha & 114 & 2.49 & 17.04 & 230 & 4130 \\
\hline 72 & Maudamani & 109 & 1.85 & 14.55 & 210 & 7740 \\
\hline 73 & Tarori Basmati & 113 & 2.01 & 15.71 & 218 & 2860 \\
\hline 74 & Mamihunger & 110 & 2.92 & 20.36 & 175 & 2640 \\
\hline 75 & Sneha & 112 & 2.31 & 20.36 & 240 & 3860 \\
\hline 76 & Savitri & 123 & 2.07 & 12.32 & 275 & 5680 \\
\hline 77 & CR Dhan 101 & 94 & 1.86 & 14.28 & 225 & 4670 \\
\hline 78 & CR Dhan 907 & 112 & 3.05 & 15.59 & 280 & 4250 \\
\hline 79 & CR Dhan 801 & 114 & 1.92 & 14.84 & 305 & 6340 \\
\hline 80 & Chinikamini & 116 & 2.46 & 15.04 & 226 & 3680 \\
\hline 81 & Nuakalajeera & 112 & 2.98 & 17.16 & 210 & 3670 \\
\hline 82 & Moti & 113 & 1.98 & 16.12 & 265 & 4760 \\
\hline 83 & Nuadhusura & 114 & 3.05 & 15.82 & 232 & 3580 \\
\hline 84 & Heera & 70 & 1.85 & 17.23 & 190 & 2940 \\
\hline
\end{tabular}


Table 1 Mean estimate values of days to 50\% flowering, grain Fe- Zn content, panicles/m² and grain yield of 102 genotypes including biofortified lines and check varieties studied during wet season, 2016 \& 2017 (Continued)

\begin{tabular}{|c|c|c|c|c|c|c|}
\hline Sl. No. & National testing No./Accession No./Name of the genotype & $\begin{array}{l}\text { Days to } 50 \% \\
\text { flowering }\end{array}$ & $\begin{array}{l}\text { Iron content } \\
\text { in ppm }\end{array}$ & $\begin{array}{l}\text { Zinc content } \\
\text { in ppm }\end{array}$ & Panicles $/ \mathrm{m}^{2}$ & $\begin{array}{l}\text { Grain } \\
\text { yield } \\
\text { (kg/ha) }\end{array}$ \\
\hline 85 & Jalmagna & 130 & 2.32 & 15.76 & 236 & 3250 \\
\hline 86 & AC44756 & 110 & 2.35 & 15.32 & 180 & 2240 \\
\hline 87 & AC44755 & 112 & 2.23 & 15.63 & 178 & 2350 \\
\hline 88 & AC44754 & 112 & 2.53 & 15.08 & 192 & 2420 \\
\hline 89 & AC44753 & 110 & 2.13 & 15.73 & 184 & 2480 \\
\hline 90 & Swarna-Sub 1 & 116 & 1.81 & 14.48 & 286 & 6130 \\
\hline 91 & Ranjit & 127 & 1.96 & 14.52 & 308 & 5620 \\
\hline 92 & Swarna & 120 & 2.03 & 14.52 & 310 & 6350 \\
\hline 93 & Jaya & 108 & 2.05 & 16.08 & 274 & 4580 \\
\hline 94 & Samalei & 110 & 2.1 & 13.16 & 262 & 4450 \\
\hline 95 & AC44752 & 112 & 1.9 & 16.24 & 186 & 2520 \\
\hline 96 & Lalat & 98 & 1.86 & 20.05 & 246 & 4520 \\
\hline 97 & MTU1010 & 97 & 1.79 & 15.04 & 258 & 4890 \\
\hline 98 & Naveen & 101 & 1.73 & 14.08 & 268 & 5120 \\
\hline 99 & Satabdi & 91 & 2.01 & 16.4 & 252 & 4460 \\
\hline 100 & Pooja & 126 & 1.73 & 13.07 & 284 & 5470 \\
\hline 101 & Sarala & 133 & 1.92 & 14.52 & 278 & 5120 \\
\hline \multirow[t]{3}{*}{102} & Agnisar & 105 & 1.75 & 13.46 & 186 & 2620 \\
\hline & $\mathrm{LSD}_{5 \%}$ & 4.551 & 0.561 & 3.140 & 59.01 & 967 \\
\hline & CV\% & 2.2 & 9.3 & 9.7 & 11.5 & 12.0 \\
\hline
\end{tabular}

The two check varieties for micronutrients, Chittimuthylu and Kalanamak had mean value of $22.3 \mathrm{mg}$ $\mathrm{kg}^{-1}$ and $25.91 \mathrm{mg} \mathrm{kg}^{-1}$ zinc in grain, respectively. In the studied panel, 56 genotypes showed moderate level of $15-20 \mathrm{mg} \mathrm{kg}^{-1}$ grain $\mathrm{Zn}$, while 32 lines exhibited low level $\left(<15 \mathrm{mg} \mathrm{kg}^{-1}\right.$ grain $\left.\mathrm{Zn}\right)$. Evaluation of 102 genotypes for grain yield and $\mathrm{Fe}-\mathrm{Zn}$ content over 2 years revealed two biofortified lines namely IET24779 and IET25465 showing at par yield with the yield check variety IR64 (Table 1). The genotypes containing high Fe or high $\mathrm{Zn}$ in milled rice and at par grain yield with yield check variety may be selected as cultivar or as donor parent for the breeding programs. The desirable genotypes with high Fe content and good grain yield were IET23829, IET 23832, BPT5204, IET24316, IET24760, IET24775, IET24779, IET25441 and IET25465. The genotypes with high $\mathrm{Zn}$ content and high grain yield were IET23824, IET24391, IET25446, IET25461, IET25465, IET25457, IET25477, Mumihunger, Sneha, Lalat and Chittimuthyalu. Genotypes IET24779, IET25465 and Chittimuthyalu produced at par yield with standard check variety IR64 along with high grain Fe-Zn content. The frequency of genotypes having low, moderate and high grain $\mathrm{Fe}$ and $\mathrm{Zn}$ content is depicted in spider graph and histogram (Fig. 1).
Relatedness among genotypes for grain yield and Fe-Zn content through genotype-by-trait biplot analysis

The scatter diagram was plotted in the first two principal components axis to generate genotype-bytrait biplot graph for grain Fe, grain $\mathrm{Zn}$ concentration and grain yield of the 102 genotypes present in the panel (Fig. 2). The first and second principal components had 87.82 and $8.08 \%$ of the total variability with eigen value of 10.18 and 0.937 , respectively (Additional file 1: Figure S1). Among the 3 traits from the principal component analysis, the grain yield contributed maximum diversity, followed by grain $\mathrm{Fe}$ content and grain $\mathrm{Zn}$ content in the panel population (Fig. 2). From the scattering pattern of genotypes in the 4 quadrants revealed the placement of the genotypes for high grain yield and Fe content in opposite direction. Higher $\mathrm{Zn}$ containing genotypes are in between grain yield and $\mathrm{Fe}$ content. However, there are some genotypes which are located nearer to origin possessing higher estimates of grain yield and grain Fe- $\mathrm{Zn}$ content. These genotypes have been encircled in the figure (Fig. 2). The top right ( $\mathrm{I}^{\text {st }}$ quadrant) and bottom right (2nd quadrant) possessed majority of the genotypes with better in $\mathrm{Fe}$ and $\mathrm{Zn}$ content in kernel along with 

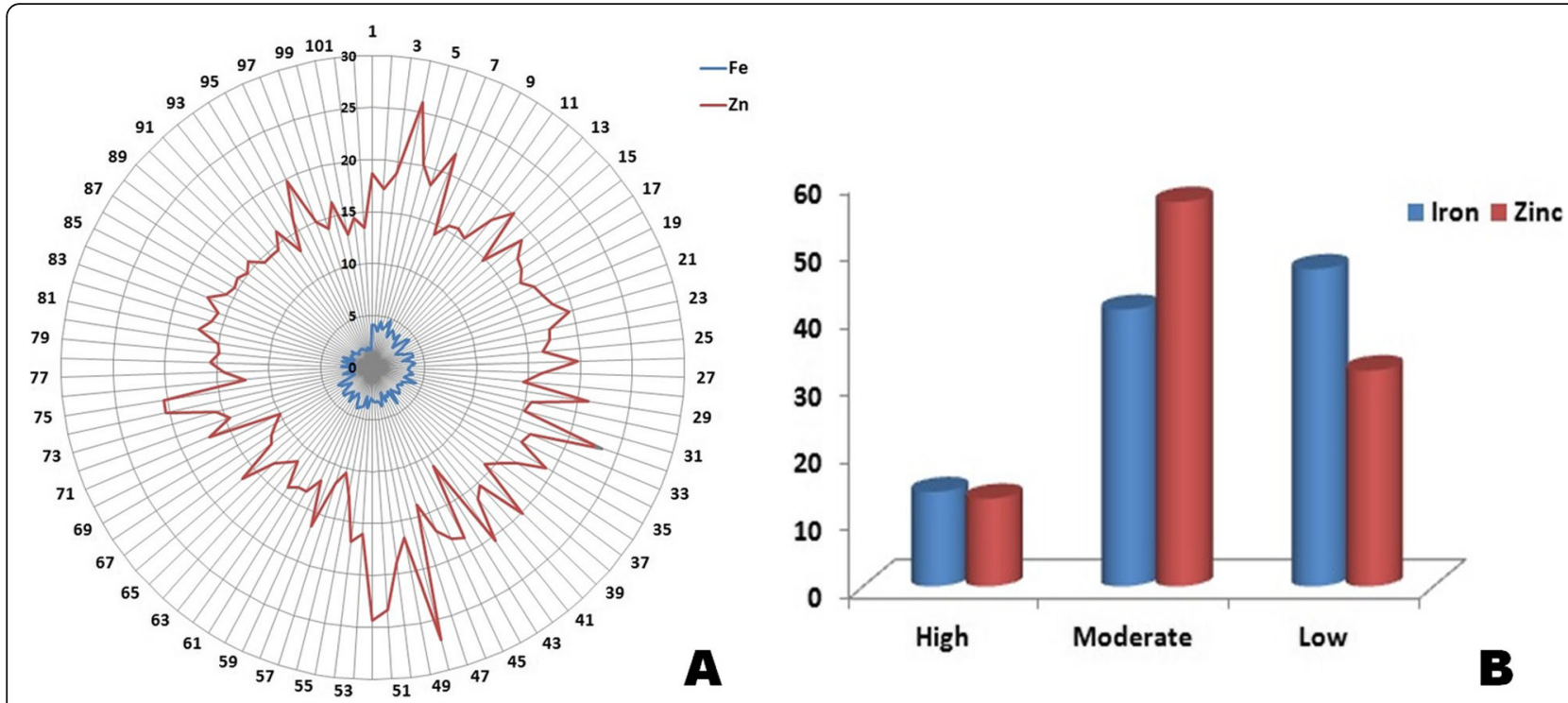

Fig. $1 \mathrm{Fe}$ and $\mathrm{Zn}$ content of 102 genotypes and their frequency distribution in the panel population. a Spider graph showing the Fe and Zn content of the genotypes. $\mathbf{b}$ Frequency of high, moderate and low Fe-Zn genotypes in the panel population

higher grain yield. The 3rd (bottom left) and 4th quadrant (top left) accommodates genotypes most of which were poor in grain $\mathrm{Fe}$ and $\mathrm{Zn}$ content with low grain yield (Fig. 2). Many genotypes with moderate in $\mathrm{Fe}$ and $\mathrm{Zn}$ content are found in 2nd quadrant. The desirable genotypes containing moderate to high grain $\mathrm{Fe}-\mathrm{Zn}$ and grain yield are located both side of the $\mathrm{X}$-axis and are encircled in the scatter diagram (Fig. 2).
Genetic diversity in the panel population using 100 molecular markers

The panel containing 102 germplasm lines exhibiting wide genetic variation for grain $\mathrm{Fe}$ and $\mathrm{Zn}$ content were genotyped using 100 molecular markers including 25 gene specific and 75 SSR markers. The loci used for genetic diversity and the calculated parameters are depicted in the table (Table 2). Two hundred forty four amplicons were obtained in toto with 2.44 average alleles per locus.

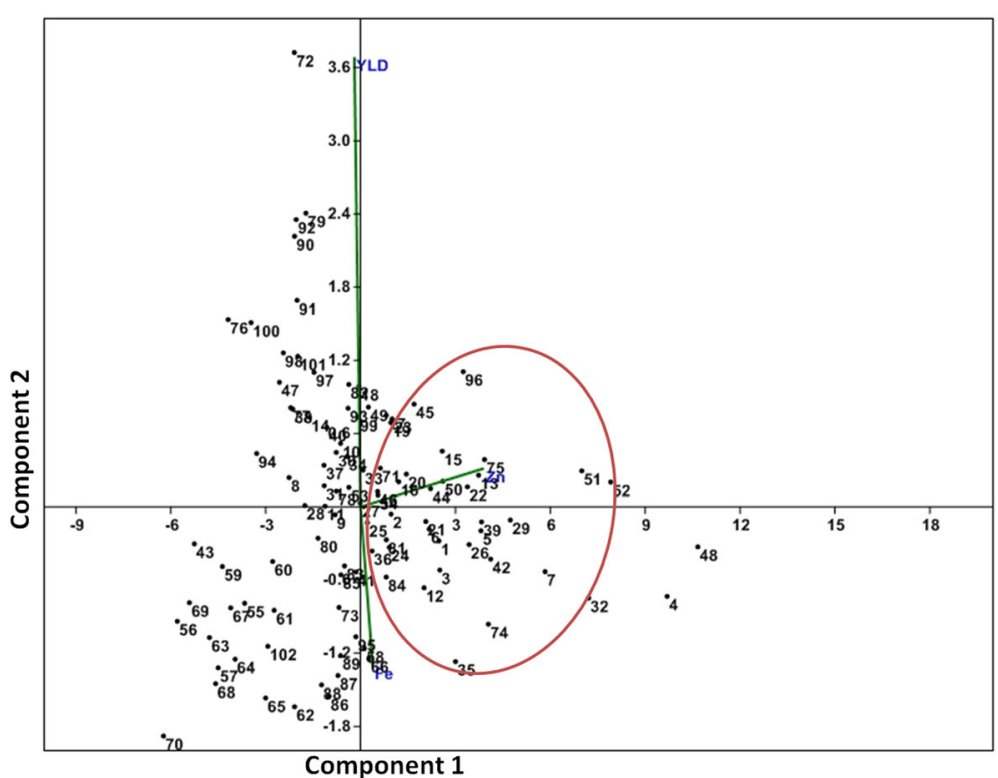

Fig. 2 Genotype-by-trait biplot graph showing 102 genotypes in two main principal components for three traits. Fe: grain iron content; Zn: grain zinc content; yld: grain yield (kg/ha); PN: panicle number; DFF: days to 50\% flowering. The dot numbers in the figure represent the serial number of the genotypes enlisted in Table 1 
Table 2 Details of 100 SSR and direct marker loci used for genotyping a panel containing 102 rice genotypes and their genetic diversity parameters

\begin{tabular}{|c|c|c|c|c|c|c|c|c|}
\hline $\begin{array}{l}\text { Sl. } \\
\text { No. }\end{array}$ & Marker Name & No. of alleles & Range of amplicon (bp) & Major allele frequency & Gene diversity & Hetero- zygosity & PIC value & $\begin{array}{l}\text { inbreeding } \\
\text { coefficient (f) }\end{array}$ \\
\hline 1 & RM243 & 3.0000 & $100-140$ & 0.6238 & 0.5331 & 0.1980 & 0.4705 & 0.6315 \\
\hline 2 & RM488 & 2.0000 & $190-210$ & 0.7344 & 0.3901 & 0.0104 & 0.3140 & 0.9736 \\
\hline 3 & RM490 & 2.0000 & $95-115$ & 0.6450 & 0.4580 & 0.0500 & 0.3531 & 0.8919 \\
\hline 4 & RM574 & 2.0000 & $150-160$ & 0.8618 & 0.2381 & 0.0132 & 0.2098 & 0.9455 \\
\hline 5 & RM122 & 2.0000 & $240-260$ & 0.6480 & 0.4562 & 0.1939 & 0.3521 & 0.5785 \\
\hline 6 & RM234 & 3.0000 & $150-160$ & 0.6050 & 0.5407 & 0.0500 & 0.4704 & 0.9084 \\
\hline 7 & RM248 & 3.0000 & $95-115$ & 0.3670 & 0.6626 & 0.1383 & 0.5884 & 0.7933 \\
\hline 8 & RM8007 & 2.0000 & $155-175$ & 0.5479 & 0.4954 & 0.1809 & 0.3727 & 0.6381 \\
\hline 9 & RM17 & 2.0000 & $150-170$ & 0.5990 & 0.4804 & 0.0521 & 0.3650 & 0.8927 \\
\hline 10 & RM260 & 2.0000 & 100-130 & 0.7407 & 0.3841 & 0.0370 & 0.3103 & 0.9053 \\
\hline 11 & RM7 & 3.0000 & $140-400$ & 0.4516 & 0.6374 & 0.0000 & 0.5625 & 1.0000 \\
\hline 12 & RM517 & 2.0000 & $250-270$ & 0.7590 & 0.3658 & 0.0000 & 0.2989 & 1.0000 \\
\hline 13 & RM501 & 2.0000 & 150-165 & 0.7611 & 0.3636 & 0.0556 & 0.2975 & 0.8488 \\
\hline 14 & OsZIP4 & 2.0000 & $310-310$ & 0.6569 & 0.4508 & 0.0000 & 0.3492 & 1.0000 \\
\hline 15 & RM594 & 2.0000 & $295-310$ & 0.8110 & 0.3066 & 0.1585 & 0.2596 & 0.4876 \\
\hline 16 & RM3412 & 3.0000 & $200-260$ & 0.5506 & 0.5936 & 0.0225 & 0.5261 & 0.9626 \\
\hline 17 & RM5638 & 2.0000 & $200-250$ & 0.5956 & 0.4817 & 0.0735 & 0.3657 & 0.8494 \\
\hline 18 & RM6712 & 3.0000 & $105-200$ & 0.6071 & 0.5541 & 0.3878 & 0.4941 & 0.3049 \\
\hline 19 & RM168 & 2.0000 & $100-120$ & 0.5700 & 0.4902 & 0.0200 & 0.3701 & 0.9596 \\
\hline 20 & RM5626 & 2.0000 & 190-210 & 0.8118 & 0.3056 & 0.0471 & 0.2589 & 0.8477 \\
\hline 21 & RM3392 & 4.0000 & $160-170$ & 0.3416 & 0.6940 & 0.4653 & 0.6320 & 0.3339 \\
\hline 22 & RM1278 & 2.0000 & $130-140$ & 0.7150 & 0.4076 & 0.1500 & 0.3245 & 0.6350 \\
\hline 23 & RM471 & 2.0000 & 110-130 & 0.7500 & 0.3750 & 0.1327 & 0.3047 & 0.6492 \\
\hline 24 & RM521 & 2.0000 & $240-270$ & 0.9278 & 0.1340 & 0.0111 & 0.1250 & 0.9180 \\
\hline 25 & RM6209 & 2.0000 & $80-90$ & 0.9798 & 0.0396 & 0.0000 & 0.0388 & 1.0000 \\
\hline 26 & RM80 & 2.0000 & $135-160$ & 0.6198 & 0.4713 & 0.2604 & 0.3602 & 0.4516 \\
\hline 27 & OsZIP8 & 2.0000 & $0-100$ & 0.6863 & 0.4306 & 0.0000 & 0.3379 & 1.0000 \\
\hline 28 & RM152 & 3.0000 & 140-180 & 0.4023 & 0.6585 & 0.5632 & 0.5845 & 0.1503 \\
\hline 29 & RM440 & 3.0000 & 150-205 & 0.7961 & 0.3438 & 0.0132 & 0.3150 & 0.9622 \\
\hline 30 & RM432 & 3.0000 & $170-350$ & 0.7990 & 0.3399 & 0.0882 & 0.3121 & 0.7426 \\
\hline 31 & RM434 & 3.0000 & $150-290$ & 0.7677 & 0.3706 & 0.0808 & 0.3233 & 0.7839 \\
\hline 32 & RM 3 & 3.0000 & $115-160$ & 0.5337 & 0.5716 & 0.0337 & 0.4871 & 0.9417 \\
\hline 33 & RM 1 & 3.0000 & $75-120$ & 0.7464 & 0.4101 & 0.0435 & 0.3730 & 0.8954 \\
\hline 34 & RM 144 & 3.0000 & $235-270$ & 0.5000 & 0.5955 & 0.1000 & 0.5137 & 0.8343 \\
\hline 35 & RM 201 & 2.0000 & $140-160$ & 0.8693 & 0.2272 & 0.0568 & 0.2014 & 0.7524 \\
\hline 36 & RM 205 & 4.0000 & $100-140$ & 0.6325 & 0.5547 & 0.0843 & 0.5172 & 0.8497 \\
\hline 37 & RM 270 & 2.0000 & $300-370$ & 0.9412 & 0.1107 & 0.0000 & 0.1046 & 1.0000 \\
\hline 38 & RM 335 & 2.0000 & $100-110$ & 0.6616 & 0.4478 & 0.0303 & 0.3475 & 0.9330 \\
\hline 39 & RM154 & 3.0000 & $130-200$ & 0.4765 & 0.6358 & 0.4471 & 0.5641 & 0.3022 \\
\hline 40 & RM211 & 3.0000 & $120-170$ & 0.6534 & 0.4884 & 0.1591 & 0.4156 & 0.6774 \\
\hline 41 & RM202 & 3.0000 & 150-200 & 0.5150 & 0.6106 & 0.0900 & 0.5385 & 0.8540 \\
\hline 42 & RM293 & 2.0000 & $200-210$ & 0.7557 & 0.3693 & 0.0114 & 0.3011 & 0.9696 \\
\hline 43 & RM85 & 3.0000 & $90-110$ & 0.5058 & 0.6182 & 0.0814 & 0.5468 & 0.8698 \\
\hline
\end{tabular}


Table 2 Details of 100 SSR and direct marker loci used for genotyping a panel containing 102 rice genotypes and their genetic diversity parameters (Continued)

\begin{tabular}{|c|c|c|c|c|c|c|c|c|}
\hline $\begin{array}{l}\text { Sl. } \\
\text { No. }\end{array}$ & Marker Name & No. of alleles & Range of amplicon (bp) & Major allele frequency & Gene diversity & Hetero- zygosity & PIC value & $\begin{array}{l}\text { inbreeding } \\
\text { coefficient (f) }\end{array}$ \\
\hline 44 & RM407 & 6.0000 & $170-550$ & 0.4892 & 0.7019 & 0.1290 & 0.6713 & 0.8180 \\
\hline 45 & RM237 & 2.0000 & $130-150$ & 0.5345 & 0.4976 & 0.0115 & 0.3738 & 0.9772 \\
\hline 46 & RM259 & 4.0000 & $180-280$ & 0.6867 & 0.4806 & 0.0400 & 0.4346 & 0.9178 \\
\hline 47 & RM421 & 2.0000 & $240-300$ & 0.9521 & 0.0912 & 0.0532 & 0.0870 & 0.4209 \\
\hline 48 & RM235 & 3.0000 & $90-150$ & 0.6462 & 0.4857 & 0.2462 & 0.4044 & 0.4990 \\
\hline 49 & RM1337 & 2.0000 & $120-140$ & 0.6875 & 0.4297 & 0.5000 & 0.3374 & \#\#\#\#\# \\
\hline 50 & RM3409 & 2.0000 & $304-340$ & 0.7692 & 0.3550 & 0.0000 & 0.2920 & 1.0000 \\
\hline 51 & RM105 & 2.0000 & $130-145$ & 0.6076 & 0.4768 & 0.0000 & 0.3632 & 1.0000 \\
\hline 52 & RM309 & 1.0000 & 190-190 & 1.0000 & 0.0000 & 0.0000 & 0.0000 & $\mathrm{NaN}$ \\
\hline 53 & RM452 & 1.0000 & $250-250$ & 1.0000 & 0.0000 & 0.0000 & 0.0000 & $\mathrm{NaN}$ \\
\hline 54 & RM204 & 3.0000 & $120-170$ & 0.4695 & 0.5741 & 0.0244 & 0.4807 & 0.9580 \\
\hline 55 & RM137 & 1.0000 & $250-250$ & 1.0000 & 0.0000 & 0.0000 & 0.0000 & $\mathrm{NaN}$ \\
\hline 56 & RM1789 & 1.0000 & 180-180 & 1.0000 & 0.0000 & 0.0000 & 0.0000 & $\mathrm{NaN}$ \\
\hline 57 & RM6641 & 2.0000 & $140-150$ & 0.7527 & 0.3723 & 0.0000 & 0.3030 & 1.0000 \\
\hline 58 & RM296 & 4.0000 & $140-410$ & 0.5337 & 0.6233 & 0.0337 & 0.5673 & 0.9465 \\
\hline 59 & RM3331 & 2.0000 & $165-175$ & 0.5306 & 0.4981 & 0.0000 & 0.3741 & 1.0000 \\
\hline 60 & RM31 & 3.0000 & $100-125$ & 0.5000 & 0.6157 & 0.0455 & 0.5419 & 0.9270 \\
\hline 61 & RM429 & 2.0000 & $120-140$ & 0.9511 & 0.0930 & 0.0326 & 0.0887 & 0.6527 \\
\hline 62 & RM556 & 2.0000 & 130-190 & 0.7157 & 0.4070 & 0.0196 & 0.3242 & 0.9523 \\
\hline 63 & RM585 & 4.0000 & $130-210$ & 0.3824 & 0.6915 & 0.0196 & 0.6330 & 0.9719 \\
\hline 64 & RM23 & 2.0000 & $150-160$ & 0.8713 & 0.2243 & 0.0000 & 0.1991 & 1.0000 \\
\hline 65 & RM34 & 2.0000 & 160-180 & 0.9188 & 0.1493 & 0.0875 & 0.1382 & 0.4191 \\
\hline 66 & RM53 & 2.0000 & $180-200$ & 0.5714 & 0.4898 & 0.0000 & 0.3698 & 1.0000 \\
\hline 67 & RM300 & 3.0000 & $130-150$ & 0.6344 & 0.5305 & 0.0000 & 0.4743 & 1.0000 \\
\hline 68 & RM315 & 2.0000 & $145-150$ & 0.8687 & 0.2281 & 0.0000 & 0.2021 & 1.0000 \\
\hline 69 & RM339 & 3.0000 & 150-190 & 0.7475 & 0.4081 & 0.0707 & 0.3706 & 0.8283 \\
\hline 70 & RM400 & 4.0000 & $250-340$ & 0.6571 & 0.5224 & 0.0000 & 0.4819 & 1.0000 \\
\hline 71 & RM528 & 2.0000 & $300-320$ & 0.5464 & 0.4957 & 0.0000 & 0.3728 & 1.0000 \\
\hline 72 & RM486 & 2.0000 & $135-140$ & 0.7526 & 0.3724 & 0.0206 & 0.3031 & 0.9452 \\
\hline 73 & RM340 & 6.0000 & $130-290$ & 0.5215 & 0.6656 & 0.1828 & 0.6293 & 0.7279 \\
\hline 74 & RM1132 & 4.0000 & 95-140 & 0.5505 & 0.6145 & 0.0303 & 0.5617 & 0.9512 \\
\hline 75 & RM441 & 1.0000 & $170-170$ & 1.0000 & 0.0000 & 0.0000 & 0.0000 & $\mathrm{NaN}$ \\
\hline 76 & RM590 & 1.0000 & 190-190 & 1.0000 & 0.0000 & 0.0000 & 0.0000 & 0.0000 \\
\hline 77 & RM258 & 1.0000 & $250-250$ & 1.0000 & 0.0000 & 0.0000 & 0.0000 & 0.0000 \\
\hline 78 & GRMM9-1 & 2.0000 & $0-275$ & 0.6961 & 0.4231 & 0.0000 & 0.3336 & 1.0000 \\
\hline 79 & GRMM9-2 & 2.0000 & $0-150$ & 0.9216 & 0.1446 & 0.0000 & 0.1341 & 1.0000 \\
\hline 80 & OsNAC & 2.0000 & $0-600$ & 0.7917 & 0.3299 & 0.0000 & 0.2755 & 1.0000 \\
\hline 81 & OsZIP8A & 2.0000 & $0-900$ & 0.5882 & 0.4844 & 0.0000 & 0.3671 & 1.0000 \\
\hline 82 & OsZIP8C & 2.0000 & $0-930$ & 0.5980 & 0.4808 & 0.0000 & 0.3652 & 1.0000 \\
\hline 83 & OsYSL4E & 3.0000 & $0-851$ & 0.5000 & 0.6250 & 0.0000 & 0.5546 & 1.0000 \\
\hline 84 & OsMTP1A & 2.0000 & $0-950$ & 0.7451 & 0.3799 & 0.0000 & 0.3077 & 1.0000 \\
\hline 85 & OsNRAMP5G & 2.0000 & $0-150$ & 0.6373 & 0.4623 & 0.0000 & 0.3555 & 1.0000 \\
\hline 86 & IRMM9-1 & 2.0000 & $0-200$ & 0.7059 & 0.4152 & 0.0000 & 0.3290 & 1.0000 \\
\hline
\end{tabular}


Table 2 Details of 100 SSR and direct marker loci used for genotyping a panel containing 102 rice genotypes and their genetic diversity parameters (Continued)

\begin{tabular}{|c|c|c|c|c|c|c|c|c|}
\hline $\begin{array}{l}\text { Sl. } \\
\text { No. }\end{array}$ & Marker Name & No. of alleles & Range of amplicon (bp) & Major allele frequency & Gene diversity & Hetero- zygosity & PIC value & $\begin{array}{l}\text { inbreeding } \\
\text { coefficient (f) }\end{array}$ \\
\hline 87 & OsYSL1 & 2.0000 & $0-230$ & 0.6667 & 0.4444 & 0.0000 & 0.3457 & 1.0000 \\
\hline 88 & OsYSL2A & 2.0000 & $0-150$ & 0.9216 & 0.1446 & 0.0000 & 0.1341 & 1.0000 \\
\hline 89 & OsYSL2B & 3.0000 & $0-330$ & 0.4412 & 0.6353 & 0.0000 & 0.5590 & 1.0000 \\
\hline 90 & OsYSL5 & 2.0000 & $0-380$ & 0.5098 & 0.4998 & 0.0000 & 0.3749 & 1.0000 \\
\hline 91 & OsYSL6 & 2.0000 & $0-166$ & 0.5098 & 0.4998 & 0.0000 & 0.3749 & 1.0000 \\
\hline 92 & OsYSL11 & 2.0000 & $0-190$ & 0.6569 & 0.4508 & 0.0000 & 0.3492 & 1.0000 \\
\hline 93 & OsZIP6A & 3.0000 & $0-170$ & 0.5098 & 0.5352 & 0.0000 & 0.4280 & 1.0000 \\
\hline 94 & OsZIP6B & 2.0000 & $0-220$ & 0.6078 & 0.4767 & 0.0000 & 0.3631 & 1.0000 \\
\hline 95 & OsZIP7 & 3.0000 & $0-180$ & 0.6569 & 0.4796 & 0.0000 & 0.4025 & 1.0000 \\
\hline 96 & OsZIP8 & 2.0000 & $0-160$ & 0.5294 & 0.4983 & 0.0000 & 0.3741 & 1.0000 \\
\hline 97 & OsNRAMP1A & 3.0000 & $0-310$ & 0.6667 & 0.4931 & 0.0000 & 0.4364 & 1.0000 \\
\hline 98 & OsNRAMP1B & 2.0000 & $0-250$ & 0.6471 & 0.4567 & 0.0000 & 0.3524 & 1.0000 \\
\hline 99 & OsFER1 & 3.0000 & $0-170$ & 0.8431 & 0.2737 & 0.0000 & 0.2518 & 1.0000 \\
\hline \multirow[t]{2}{*}{100} & OsFER2 & 3.0000 & $0-475$ & 0.4804 & 0.5992 & 0.0000 & 0.5157 & 1.0000 \\
\hline & Mean & 2.44 & & 0.682038 & 0.414195 & 0.061385 & 0.348211 & 0.849347 \\
\hline
\end{tabular}

The number of alleles ranged from 1 to 6 per marker with RM340 showing the highest number of alleles in the panel genotypes. The average major allele frequency of $\mathrm{Fe}$ and $\mathrm{Zn}$ linked polymorphic markers was observed to be 0.675 ranging within the bracket of 0.4023 (RM152) and 1.0000 (RM309, RM452, RM137, RM1789 and RM441) (Table 2). The PIC value ranged from 0.000 (RM6209, sZIP8, RM3409, RM309, RM452, RM137, RM1789, RM556, RM23, RM34, RM441 and GRMM92) to 0.6713 (RM407) with mean PIC of 0.3553 . The observed average heterozygosity (Ho) was 0.0496 with a range of $0.00-0.6714$. Amongst the markers used, only 36 showed heterogygocity value to be more than zero, whereas 64 exhibited zero values. The average gene diversity (He) varied from 0.0000 (RM309, RM452, RM137, RM1789, RM44, RM590 and RM258) to 0.6711 (RM3392) with an average value of 0.35 .

\section{Population structure}

A Bayesian clustering approach was followed for estimation of genetic structure by taking probable subpopulations $(\mathrm{K})$ and higher delta K-value using the STRUCTURE 2.3.6 software. The genotypes in the panel exhibiting variation for grain Fe and $\mathrm{Zn}$ content were evaluated for genetic structure following. It categorized the genotypes into two sub-populations (Fig. 3a, b) with a high $\Delta \mathrm{K}$ peak value of 495.2 at $\mathrm{K}=2$ among the assumed $\mathrm{K}$ (Fig. 3a). But, the classification was not robust with respect to $\mathrm{Fe}-\mathrm{Zn}$ content in the genotypes. However, at $\mathrm{K}=3$ with $\Delta \mathrm{K}$ value of 117.9 categorized the population into three distinct sub-populations with perfect grouping. At $\mathrm{K}=3$, the sub-population 1 (SP1), sub-population 2 (SP2) and sub-population 3 (SP3) included 18, 44 and 40 genotypes, respectively whereas eleven genotypes were admixture type having major genetic constituent from three sub-groups (Fig. 3c; Table 3). The SP1 and SP2 included majority of moderate and high grain Fe- $\mathrm{Zn}$ containing genotypes accommodating $100 \%$ of total high $\mathrm{Fe}\left(>4 \mathrm{mg} \mathrm{kg}^{-1}\right)$ genotypes, $97.3 \%$ of total moderate $\mathrm{Fe}\left(3-4 \mathrm{mg} \mathrm{kg}^{-1}\right)$ genotypes, $90 \%$ of total high $\mathrm{Zn}\left(>20 \mathrm{mg} \mathrm{kg}^{-1}\right.$ ) genotypes and $69.2 \%$ of total moderate $\mathrm{Zn}\left(15-20 \mathrm{mg} \mathrm{kg}^{-1}\right)$ genotypes. Maximum allele frequency divergence between the two subpopulations (net nucleotide distance) 1-2, 1-3 and $2-3$ were $0.1239,0.1813$ and 0.2480 , respectively. The average distance (expected heterozygosity) between individuals in cluster 1 , cluster 2 and cluster 3 were $0.3140,0.3051$ and 0.3115 , respectively. The three sub-populations showed fixation index values $\left(\mathrm{F}_{\mathrm{ST}}\right)$ of 0.287 for SP1, 0.385 for SP2 and 0.4853 for SP3. A lower value of alpha (alpha $=0.0388$ ) was found for the studied panel population. The distribution pattern of alpha-value in the panel population showed a leptokurtic symmetry while distribution of $\mathrm{F}_{\mathrm{ST}}$ values in the sub-populations were almost in symmetric shape with same to the left and right from the centre (Additional file 2: Figure S2). Further at $\mathrm{K}=7$ with $\Delta \mathrm{K}$ value of 3.946, the software categorized the entire population into 7 distinct sub-populations classifying the population into more subtle classes according to grain $\mathrm{Fe}-\mathrm{Zn}$ content. 

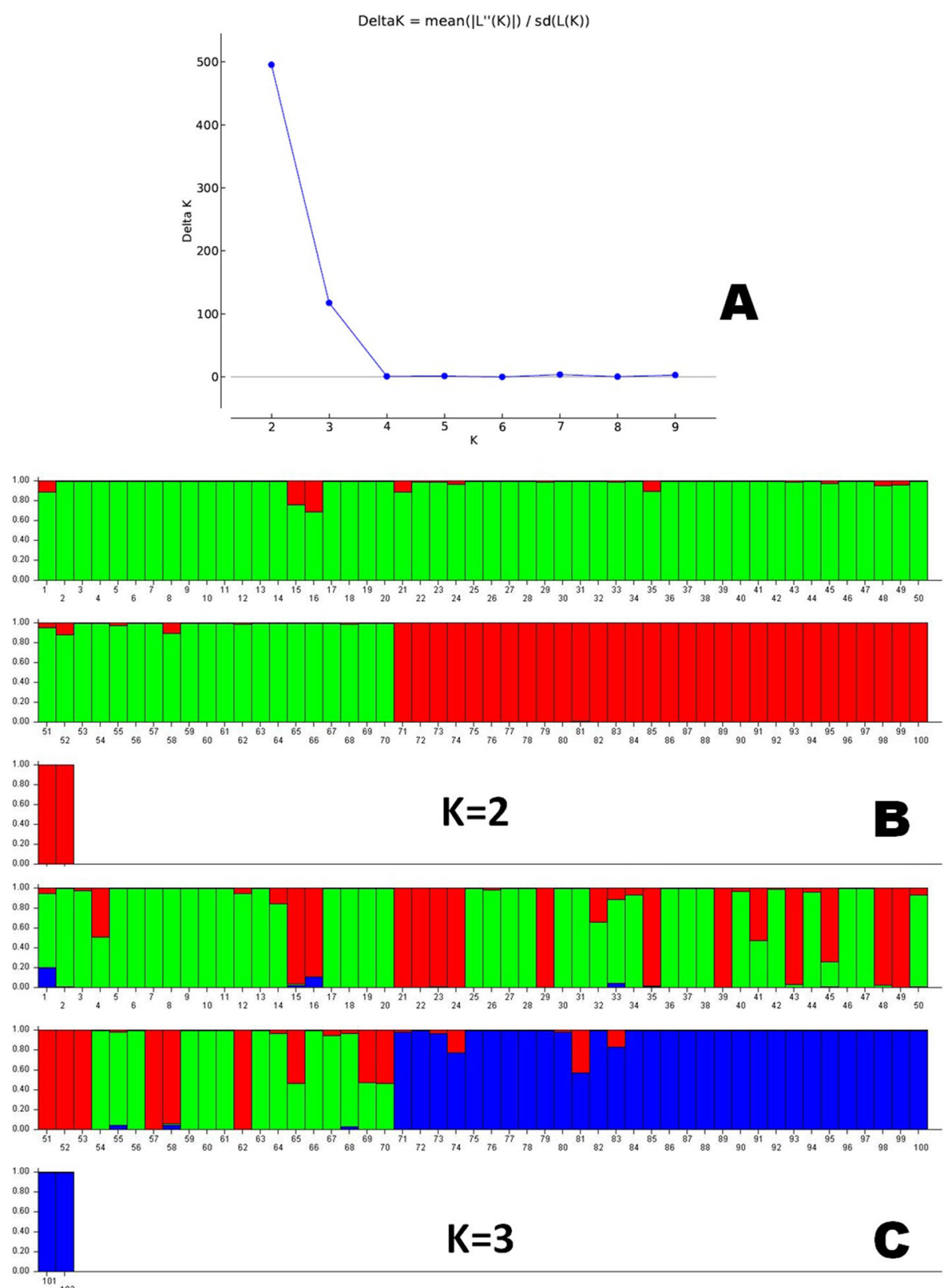

Fig. 3 a Graph of delta $\mathrm{K}$ value, an ad-hoc statistic related to the rate of change in the log probability of data between successive $\mathrm{K}$ values; $\mathbf{b}$ Population structure of the 102-panel population placed based on membership probability fractions of individual genotypes at $\mathrm{K}=2$ and $\mathbf{c}$ Population structure of the 102-panel population placed based on membership probability fractions of individual genotypes at $\mathrm{K}=3$. The genotypes with the probability of $\geq 80 \%$ membership fractions were assigned to corresponding subgroups with others categorized as admixture. The numbers in the figure represent the serial number of the genotypes enlisted in Table 1

\section{Analysis of molecular variance (AMOVA) and LD decay plot}

The analysis of molecular variance (AMOVA) showed genetic variations between and within the subpopulations at $\mathrm{K}=2, \mathrm{~K}=3$ and $\mathrm{K}=7$ (Table 4). The genetic variations between and within the two subpopulations $(K=2)$ was $41 \%$ among the populations, $50 \%$ among individuals and $9 \%$ variation within individuals in the panel population. At 3 subpopulations, $38 \%$ of the variation among populations, $52 \%$ among individuals and $10 \%$ variation within individuals in the panel population. But, the analysis at $\mathrm{K}=7$ revealed $39 \%$ of the variation among populations, $51 \%$ among individuals and $10 \%$ variation within individuals resulted from the analysis. Wright's F statistic was used to calculate the deviation from Hardy-Weinberg's 
Table 3 The inferred ancestry value and population structure in the panel population containing 102 genotypes with their Fe-Zn classification category

\begin{tabular}{|c|c|c|c|c|c|c|c|c|c|}
\hline \multirow{2}{*}{$\begin{array}{l}\text { Sl. } \\
\text { No. }\end{array}$} & \multirow{2}{*}{$\begin{array}{l}\text { Genotype } \\
\text { name/National } \\
\text { testing No. }\end{array}$} & \multicolumn{2}{|c|}{ Inferred ancestry at $\mathrm{K}=2$} & \multirow{2}{*}{$\begin{array}{l}\text { Structure } \\
\text { group }\end{array}$} & \multicolumn{3}{|c|}{ Inferred ancestry at $\mathrm{K}=3$} & \multirow[t]{2}{*}{ group } & \multirow{2}{*}{$\begin{array}{l}\text { Classification } \\
\text { of genotype } \\
\text { based on Fe \& } \\
\text { Zn content }\end{array}$} \\
\hline & & Q1 & Q2 & & Q1 & Q2 & Q3 & & \\
\hline 1 & IET23829 & 0.111 & 0.889 & SP2 & 0.053 & 0.745 & 0.202 & SP2 & $\mathrm{H}-\mathrm{Fe} \& \mathrm{M}-\mathrm{Zn}$ \\
\hline 2 & IR64 & 0.004 & 0.996 & SP2 & 0.006 & 0.985 & 0.009 & SP2 & $\mathrm{H}-\mathrm{Fe} \& \mathrm{M}-\mathrm{Zn}$ \\
\hline 3 & IET23834 & 0.001 & 0.999 & SP2 & 0.024 & 0.975 & 0.001 & SP2 & M-Fe \& M-Zn \\
\hline 4 & Kalanamak & 0.001 & 0.999 & SP2 & 0.489 & 0.51 & 0.001 & SP2 & $\mathrm{H}-\mathrm{Fe} \& \mathrm{H}-\mathrm{Zn}$ \\
\hline 5 & IET23824 & 0.001 & 0.999 & SP2 & 0.006 & 0.993 & 0.001 & SP2 & M-Fe \& H-Zn \\
\hline 6 & IET23832 & 0.002 & 0.998 & SP2 & 0.001 & 0.998 & 0.001 & SP2 & $\mathrm{H}-\mathrm{Fe} \& \mathrm{M}-\mathrm{Zn}$ \\
\hline 7 & Chittimuthyalu & 0.001 & 0.999 & SP2 & 0.002 & 0.998 & 0.001 & SP2 & $\mathrm{H}-\mathrm{Fe} \& \mathrm{H}-\mathrm{Zn}$ \\
\hline 8 & IET24780 & 0.001 & 0.999 & SP2 & 0.001 & 0.998 & 0.001 & SP2 & $\mathrm{H}-\mathrm{Fe} \& \mathrm{H}-\mathrm{Zn}$ \\
\hline 9 & ВРT5204 & 0.001 & 0.999 & SP2 & 0.007 & 0.992 & 0.001 & SP2 & $\mathrm{H}-\mathrm{Fe} \& \mathrm{M}-\mathrm{Zn}$ \\
\hline 10 & IET24771 & 0.001 & 0.999 & SP2 & 0.002 & 0.997 & 0.001 & SP2 & $M-F e ~ \& ~ M-Z n$ \\
\hline 11 & IET24766 & 0.001 & 0.999 & SP2 & 0.005 & 0.994 & 0.001 & SP2 & $M-F e ~ \& ~ M-Z n$ \\
\hline 12 & IET24316 & 0.001 & 0.999 & SP2 & 0.057 & 0.938 & 0.004 & SP2 & $\mathrm{H}-\mathrm{Fe} \& \mathrm{M}-\mathrm{Zn}$ \\
\hline 13 & IET24391 & 0.001 & 0.999 & SP2 & 0.002 & 0.997 & 0.001 & SP2 & L-Fe \& H-Zn \\
\hline 14 & IET24777 & 0.002 & 0.998 & SP2 & 0.154 & 0.843 & 0.003 & SP2 & L-Fe \& L-Zn \\
\hline 15 & IET24760 & 0.242 & 0.758 & SP2 & 0.96 & 0.018 & 0.022 & SP1 & $\mathrm{H}-\mathrm{Fe} \& \mathrm{M}-\mathrm{Zn}$ \\
\hline 16 & IET24775 & 0.311 & 0.689 & SP2 & 0.886 & 0.005 & 0.108 & SP1 & $\mathrm{H}-\mathrm{Fe} \& \mathrm{M}-\mathrm{Zn}$ \\
\hline 17 & IET24783 & 0.002 & 0.998 & SP2 & 0.002 & 0.997 & 0.001 & SP2 & $M-F e ~ \& ~ M-Z n$ \\
\hline 18 & IET24544 & 0.001 & 0.999 & SP2 & 0.003 & 0.996 & 0.002 & SP2 & L-Fe \& M-Zn \\
\hline 19 & IET24336 & 0.001 & 0.999 & SP2 & 0.005 & 0.994 & 0.001 & SP2 & L-Fe \& M-Zn \\
\hline 20 & IET24772 & 0.001 & 0.999 & SP2 & 0.003 & 0.996 & 0.001 & SP2 & $M-F e ~ \& ~ M-Z n$ \\
\hline 21 & IET24557 & 0.111 & 0.889 & SP2 & 0.993 & 0.002 & 0.004 & SP1 & $M-F e ~ \& ~ M-Z n$ \\
\hline 22 & IET24779 & 0.009 & 0.991 & SP2 & 0.997 & 0.002 & 0.001 & SP1 & $\mathrm{H}-\mathrm{Fe} \& \mathrm{M}-\mathrm{Zn}$ \\
\hline 23 & IET24774 & 0.008 & 0.992 & SP2 & 0.99 & 0.009 & 0.001 & SP1 & L-Fe \& M-Zn \\
\hline 24 & IET24787 & 0.032 & 0.968 & SP2 & 0.996 & 0.002 & 0.002 & SP1 & $M-F e ~ \& ~ M-Z n$ \\
\hline 25 & IET25441 & 0.001 & 0.999 & SP2 & 0.004 & 0.994 & 0.001 & SP2 & $\mathrm{H}$-Fe \& M-Zn \\
\hline 26 & IET25443 & 0.001 & 0.999 & SP2 & 0.015 & 0.984 & 0.001 & SP2 & $M-F e ~ \& ~ M-Z n$ \\
\hline 27 & IET25444 & 0.001 & 0.999 & SP2 & 0.001 & 0.998 & 0.001 & SP2 & $M-F e ~ \& ~ M-Z n$ \\
\hline 28 & IET25445 & 0.001 & 0.999 & SP2 & 0.002 & 0.997 & 0.001 & SP2 & M-Fe \& L-Zn \\
\hline 29 & IET25446 & 0.009 & 0.991 & SP2 & 0.995 & 0.003 & 0.002 & SP1 & M-Fe \& H-Zn \\
\hline 30 & IET25447 & 0.001 & 0.999 & SP2 & 0.002 & 0.997 & 0.001 & SP2 & $M-F e ~ \& M-Z n$ \\
\hline 31 & IET25449 & 0.001 & 0.999 & SP2 & 0.001 & 0.998 & 0.001 & SP2 & $M-F e ~ \& ~ M-Z n$ \\
\hline 32 & IET25450 & 0.001 & 0.999 & SP2 & 0.341 & 0.658 & 0.002 & SP2 & $\mathrm{H}-\mathrm{Fe} \& \mathrm{H}-\mathrm{Zn}$ \\
\hline 33 & IET25452 & 0.012 & 0.988 & SP2 & 0.112 & 0.843 & 0.045 & SP2 & $M-F e ~ \& ~ M-Z n$ \\
\hline 34 & IET25453 & 0.001 & 0.999 & SP2 & 0.067 & 0.93 & 0.003 & SP2 & $M-F e ~ \& ~ M-Z n$ \\
\hline 35 & IET25454 & 0.106 & 0.894 & SP2 & 0.983 & 0.007 & 0.01 & SP1 & $M-F e ~ \& ~ M-Z n$ \\
\hline 36 & IET25457 & 0.001 & 0.999 & SP2 & 0.002 & 0.997 & 0.001 & SP2 & L-Fe \& M-Zn \\
\hline 37 & IET25459 & 0.001 & 0.999 & SP2 & 0.002 & 0.998 & 0.001 & SP2 & L-Fe \& M-Zn \\
\hline 38 & IET25460 & 0.001 & 0.999 & SP2 & 0.004 & 0.995 & 0.001 & SP2 & M-Fe \& L-Zn \\
\hline 39 & IET25461 & 0.007 & 0.993 & SP2 & 0.994 & 0.005 & 0.001 & SP1 & M-Fe \& H-Zn \\
\hline 40 & IET25463 & 0.002 & 0.998 & SP2 & 0.032 & 0.965 & 0.004 & SP2 & $M-F e ~ \& ~ M-Z n$ \\
\hline 41 & IET25464 & 0.001 & 0.999 & SP2 & 0.525 & 0.474 & 0.001 & SP1 & L-Fe \& M-Zn \\
\hline
\end{tabular}


Table 3 The inferred ancestry value and population structure in the panel population containing 102 genotypes with their Fe-Zn classification category (Continued)

\begin{tabular}{|c|c|c|c|c|c|c|c|c|c|}
\hline \multirow{2}{*}{$\begin{array}{l}\text { Sl. } \\
\text { No. }\end{array}$} & \multirow{2}{*}{$\begin{array}{l}\text { Genotype } \\
\text { name/National } \\
\text { testing No. }\end{array}$} & \multicolumn{2}{|c|}{ Inferred ancestry at $\mathrm{K}=2$} & \multirow{2}{*}{$\begin{array}{l}\text { Structure } \\
\text { group }\end{array}$} & \multicolumn{3}{|c|}{ Inferred ancestry at $\mathrm{K}=3$} & \multirow[t]{2}{*}{ group } & \multirow{2}{*}{$\begin{array}{l}\text { Classification } \\
\text { of genotype } \\
\text { based on Fe \& } \\
\text { Zn content }\end{array}$} \\
\hline & & Q1 & Q2 & & Q1 & Q2 & Q3 & & \\
\hline 42 & IET25465 & 0.001 & 0.999 & SP2 & 0.009 & 0.988 & 0.002 & SP2 & $\mathrm{H}-\mathrm{Fe} \& \mathrm{H}-\mathrm{Zn}$ \\
\hline 43 & Gontra Bidhan3 & 0.009 & 0.991 & SP2 & 0.97 & 0.028 & 0.001 & SP1 & $L-F e ~ \& ~ L-Z n$ \\
\hline 44 & IET25469 & 0.001 & 0.999 & SP2 & 0.038 & 0.959 & 0.003 & SP2 & $M-F e ~ \& ~ M-Z n$ \\
\hline 45 & IET25470 & 0.022 & 0.978 & SP2 & 0.736 & 0.256 & 0.008 & SP1 & $L-F e ~ \& M-Z n$ \\
\hline 46 & IET25471 & 0.001 & 0.999 & SP2 & 0.004 & 0.994 & 0.002 & SP2 & $M-F e ~ \& M-Z n$ \\
\hline 47 & DRRH3 & 0.001 & 0.999 & SP2 & 0.004 & 0.992 & 0.003 & SP2 & $L-F e ~ \& ~ L-Z n$ \\
\hline 48 & IET25472 & 0.048 & 0.952 & SP2 & 0.976 & 0.017 & 0.007 & SP1 & M-Fe \& H-Zn \\
\hline 49 & IET25473 & 0.042 & 0.958 & SP2 & 0.996 & 0.002 & 0.002 & SP1 & $M-F e ~ \& M-Z n$ \\
\hline 50 & IET25474 & 0.002 & 0.998 & SP2 & 0.065 & 0.926 & 0.008 & SP2 & $M-F e ~ \& ~ M-Z n$ \\
\hline 51 & IET25475 & 0.05 & 0.95 & SP2 & 0.997 & 0.002 & 0.001 & SP1 & $\mathrm{M}-\mathrm{Fe} \& \mathrm{H}-\mathrm{Zn}$ \\
\hline 52 & IET25477 & 0.121 & 0.879 & SP2 & 0.995 & 0.001 & 0.003 & SP1 & M-Fe \& H-Zn \\
\hline 53 & IET25478 & 0.006 & 0.994 & SP2 & 0.998 & 0.001 & 0.001 & SP1 & L-Fe \& M-Zn \\
\hline 54 & IET25479 & 0.001 & 0.999 & SP2 & 0.006 & 0.993 & 0.001 & SP2 & M-Fe \& M-Zn \\
\hline 55 & Lalmeeta & 0.022 & 0.978 & SP2 & 0.019 & 0.934 & 0.047 & SP2 & $L-F e ~ \& ~ L-Z n$ \\
\hline 56 & Abhimanyu & 0.001 & 0.999 & SP2 & 0.003 & 0.995 & 0.001 & SP2 & M-Fe \& L-Zn \\
\hline 57 & Kalobhutia & 0.007 & 0.993 & SP2 & 0.998 & 0.001 & 0.001 & SP1 & M-Fe \& L-Zn \\
\hline 58 & Sadakajam & 0.103 & 0.897 & SP2 & 0.94 & 0.016 & 0.044 & SP1 & $\mathrm{H}-\mathrm{Fe} \& \mathrm{M}-\mathrm{Zn}$ \\
\hline 59 & Geetanjali & 0.001 & 0.999 & SP2 & 0.002 & 0.998 & 0.001 & SP2 & M-Fe \& L-Zn \\
\hline 60 & Kakhru & 0.001 & 0.999 & SP2 & 0.001 & 0.998 & 0.001 & SP2 & L-Fe \& L-Zn \\
\hline 61 & Boanti & 0.001 & 0.999 & SP2 & 0.001 & 0.998 & 0.001 & SP2 & M-Fe \& L-Zn \\
\hline 62 & Tulsimukul & 0.008 & 0.992 & SP2 & 0.998 & 0.002 & 0.001 & SP1 & M-Fe \& L-Zn \\
\hline 63 & Kokilpatri & 0.001 & 0.999 & SP2 & 0.001 & 0.998 & 0.001 & SP2 & $L-F e ~ \& ~ L-Z n$ \\
\hline 64 & Basmatikarnal & 0.001 & 0.999 & SP2 & 0.034 & 0.965 & 0.001 & SP2 & M-Fe \& L-Zn \\
\hline 65 & Kalonunia & 0.001 & 0.999 & SP2 & 0.534 & 0.465 & 0.001 & SP1 & M-Fe \& L-Zn \\
\hline 66 & SafedLuchai2 & 0.001 & 0.999 & SP2 & 0.001 & 0.998 & 0.001 & SP2 & $M-F e ~ \& M-Z n$ \\
\hline 67 & Bankra & 0.001 & 0.999 & SP2 & 0.051 & 0.946 & 0.003 & SP2 & L-Fe \&L-Zn \\
\hline 68 & Moongi & 0.009 & 0.991 & SP2 & 0.031 & 0.935 & 0.033 & SP2 & L-Fe \& L-Zn \\
\hline 69 & Swarnakranti & 0.001 & 0.999 & SP2 & 0.524 & 0.475 & 0.001 & SP1 & M-Fe \& L-Zn \\
\hline 70 & Kalojeera & 0.001 & 0.999 & SP2 & 0.53 & 0.47 & 0.001 & SP1 & M-Fe \& L-Zn \\
\hline 71 & Ketekijoha & 0.999 & 0.001 & SP1 & 0.015 & 0.001 & 0.984 & SP3 & $L-F e \& M-Z n$ \\
\hline 72 & Maudamani & 0.999 & 0.001 & SP1 & 0.002 & 0.001 & 0.997 & SP3 & L- Fe \& L- Zn \\
\hline 73 & Tarori Basmati & 0.999 & 0.001 & SP1 & 0.032 & 0.001 & 0.967 & SP3 & L- Fe \& M- Zn \\
\hline 74 & Mamihunger & 0.998 & 0.002 & SP1 & 0.226 & 0.001 & 0.773 & SP3 & L- Fe \& H- Zn \\
\hline 75 & Sneha & 0.999 & 0.001 & SP1 & 0.002 & 0.001 & 0.998 & SP3 & L-Fe \& H-Zn \\
\hline 76 & Savitri & 0.999 & 0.001 & SP1 & 0.002 & 0.001 & 0.997 & SP3 & $L F e \& L-Z n$ \\
\hline 77 & CR Dhan 101 & 0.999 & 0.001 & SP1 & 0.003 & 0.001 & 0.996 & SP3 & L-Fe \& L-Zn \\
\hline 78 & CR Dhan 907 & 0.999 & 0.001 & SP1 & 0.001 & 0.001 & 0.998 & SP3 & $M-F e \& M-Z n$ \\
\hline 79 & CR Dhan 801 & 0.999 & 0.001 & SP1 & 0.001 & 0.001 & 0.998 & SP3 & L- Fe \& L-Zn \\
\hline 80 & Chinikamini & 0.999 & 0.001 & SP1 & 0.015 & 0.001 & 0.984 & SP3 & L- Fe \& M-Zn \\
\hline 81 & Nuakalajeera & 0.989 & 0.011 & SP1 & 0.422 & 0.001 & 0.577 & SP3 & L- Fe \& M-Zn \\
\hline 82 & Moti & 0.999 & 0.001 & SP1 & 0.002 & 0.001 & 0.997 & SP3 & $L-F e \& M-Z n$ \\
\hline
\end{tabular}


Table 3 The inferred ancestry value and population structure in the panel population containing 102 genotypes with their Fe-Zn classification category (Continued)

\begin{tabular}{|c|c|c|c|c|c|c|c|c|c|}
\hline \multirow{2}{*}{$\begin{array}{l}\text { Sl. } \\
\text { No. }\end{array}$} & \multirow{2}{*}{$\begin{array}{l}\text { Genotype } \\
\text { name/National } \\
\text { testing No. }\end{array}$} & \multicolumn{2}{|c|}{ Inferred ancestry at $\mathrm{K}=2$} & \multirow{2}{*}{$\begin{array}{l}\text { Structure } \\
\text { group }\end{array}$} & \multicolumn{3}{|c|}{ Inferred ancestry at $\mathrm{K}=3$} & \multirow[t]{2}{*}{ group } & \multirow{2}{*}{$\begin{array}{l}\text { Classification } \\
\text { of genotype } \\
\text { based on Fe } 8 \\
\text { Zn content }\end{array}$} \\
\hline & & $\overline{\mathrm{Q} 1}$ & Q2 & & $\mathrm{Q1}$ & Q2 & Q3 & & \\
\hline 83 & Nuadhusura & 0.999 & 0.001 & SP1 & 0.169 & 0.001 & 0.83 & SP3 & $M-F e \& M-Z n$ \\
\hline 84 & Heera & 0.999 & 0.001 & SP1 & 0.002 & 0.001 & 0.997 & SP3 & L- Fe \& M-Zn \\
\hline 85 & Jalmagna & 0.999 & 0.001 & SP1 & 0.002 & 0.001 & 0.997 & SP3 & L- Fe \& M-Zn \\
\hline 86 & AC44756 & 0.999 & 0.001 & SP1 & 0.001 & 0.001 & 0.998 & SP3 & L- Fe \& M-Zn \\
\hline 87 & AC44755 & 0.999 & 0.001 & SP1 & 0.001 & 0.001 & 0.998 & SP3 & L- Fe \& M-Zn \\
\hline 88 & AC44754 & 0.999 & 0.001 & SP1 & 0.001 & 0.001 & 0.998 & SP3 & L- Fe \& M-Zn \\
\hline 89 & AC44753 & 0.999 & 0.001 & SP1 & 0.003 & 0.001 & 0.996 & SP3 & $L-F e \& M-Z n$ \\
\hline 90 & Swarna-Sub 1 & 0.999 & 0.001 & SP1 & 0.004 & 0.001 & 0.995 & SP3 & L-Fe \& M- Zn \\
\hline 91 & Ranjit & 0.999 & 0.001 & SP1 & 0.002 & 0.001 & 0.997 & SP3 & L- Fe \& L- Zn \\
\hline 92 & Swarna & 0.999 & 0.001 & SP1 & 0.001 & 0.001 & 0.998 & SP3 & $L-F \& L-Z n$ \\
\hline 93 & Jaya & 0.999 & 0.001 & SP1 & 0.002 & 0.001 & 0.998 & SP3 & L-Fe \& M- Zn \\
\hline 94 & Samalei & 0.999 & 0.001 & SP1 & 0.001 & 0.001 & 0.998 & SP3 & L- Fe \& L- Zn \\
\hline 95 & AC44752 & 0.999 & 0.001 & SP1 & 0.001 & 0.001 & 0.998 & SP3 & L- Fe \& M- Zn \\
\hline 96 & Lalat & 0.999 & 0.001 & SP1 & 0.001 & 0.001 & 0.998 & SP3 & L- Fe \& H- Zn \\
\hline 97 & MTU1010 & 0.999 & 0.001 & SP1 & 0.004 & 0.001 & 0.995 & SP3 & $L-F e \& M-Z n$ \\
\hline 98 & Naveen & 0.999 & 0.001 & SP1 & 0.002 & 0.001 & 0.997 & SP3 & L- Fe \& L- Zn \\
\hline 99 & Satabdi & 0.999 & 0.001 & SP1 & 0.001 & 0.001 & 0.999 & SP3 & L- Fe \& M- Zn \\
\hline 100 & Pooja & 0.999 & 0.001 & SP1 & 0.001 & 0.001 & 0.998 & SP3 & L- Fe \& L- Zn \\
\hline 101 & Sarala & 0.999 & 0.001 & SP1 & 0.002 & 0.001 & 0.997 & SP3 & L- Fe \& L- Zn \\
\hline 102 & Agnisar & 0.999 & 0.001 & SP1 & 0.001 & 0.001 & 0.998 & SP3 & L- Fe \& L- Zn \\
\hline
\end{tabular}

L-Fe Low grain Fe content, $M$-Fe Medium grain Fe content, $H$-Fe High grain Fe content, L-Zn: L-Zn Low grain Zn content, $M$-Zn Medium grain Zn content, $H$-Zn High grain $\mathrm{Zn}$ content

prediction. The $\mathrm{F}_{\mathrm{IS}}$ and $\mathrm{F}_{\mathrm{IT}}$ value for all the 100 loci were 0.844 and 0.904 , whereas $\mathrm{F}_{\mathrm{ST}}$ was 0.385 among populations at three sub-populations analysis. The values of $F_{S T}, F_{I S}$ and $F_{I T}$ for 7 sub-population level were 0.386 , 0.831 and 0.897 , respectively. The $\mathrm{F}_{\mathrm{ST}}$ values at both
$\mathrm{K}=3$ and $\mathrm{K}=7$ could discriminate the sub-populations from each other revealing differences among themselves. A moderate linearized $\mathrm{F}_{\mathrm{ST}}$ value of 0.385 at $\mathrm{K}=3$ and 0.386 at $K=7$ were computed for the sub-populations. The $F_{S T}$ values of each sub-population and their

Table 4 Analysis of molecular variance (AMOVA) of the sub-populations of panel population at $\mathrm{K}=2$ and $\mathrm{K}=3$ for Fe and $\mathrm{Zn}$ content in milled rice of 102 genotypes

\begin{tabular}{|c|c|c|c|c|c|c|c|c|}
\hline \multirow[t]{2}{*}{ Source of variation } & \multicolumn{4}{|c|}{ AMOVA for the three sub-populations at $\mathrm{K}=2$} & \multicolumn{4}{|c|}{ AMOVA for the seven sub-populations at $\mathrm{K}=3$} \\
\hline & df. & $\begin{array}{l}\text { Mean sum of } \\
\text { squares }\end{array}$ & $\begin{array}{l}\text { Variance } \\
\text { components }\end{array}$ & $\begin{array}{l}\text { Percentage } \\
\text { variation }\end{array}$ & $\mathrm{df}$. & $\begin{array}{l}\text { Mean sum } \\
\text { of squares }\end{array}$ & $\begin{array}{l}\text { Variance } \\
\text { components }\end{array}$ & $\begin{array}{l}\text { Percentage } \\
\text { variation }\end{array}$ \\
\hline Among populations & 1 & 1192.43 & 13.175 & 41 & 2 & 743 & 10.99 & 38 \\
\hline Among individuals (accessions) within population & 100 & 35.06 & 16.163 & 50 & 99 & 32.45 & 14.86 & 52 \\
\hline Within individuals (accessions) & 102 & 2.74 & 2.74 & 9 & 102 & 2.74 & 2.74 & 10 \\
\hline Total & 203 & & & 100 & 203 & & 28.59 & 100 \\
\hline F-Statistics & Value & $P$-value & & & Value & $P$-value & & \\
\hline $\mathrm{F}_{\mathrm{ST}}$ & 0.411 & 0.001 & & & 0.3858 & 0.001 & & \\
\hline$F_{1 S}$ & 0.855 & 0.001 & & & 0.844 & 0.001 & & \\
\hline $\mathrm{F}_{\mathrm{IT}}$ & 0.915 & 0.001 & & & 0.904 & 0.001 & & \\
\hline $\mathrm{F}_{\mathrm{ST}} \max$ & 0.614 & & & & 0.640 & & & \\
\hline $\mathrm{F}_{\mathrm{ST}}^{\prime}$ & 0.669 & & & & 0.601 & & & \\
\hline
\end{tabular}


distribution pattern showed a clear differentiation among the sub-populations from each other (Additional file 2: Figure S2).

The linkage disequilibrium decay rate is crucial for maintenance of disequilibrium in a population. This provides scope for mapping of complex traits through marker-trait association. Linkage disequilibrium plot was generated for a population by plotting Syntenic $r 2$ values against the physical distance in million base pair. A sharp decline in LD decay was obtained for the linked markers at 1-2 $\mathrm{M}$ base pair and subsequently showed a very slow and gradual decay (Fig. 4).

\section{Grouping of panel population by principal coordinates and cluster analyses}

Principal coordinate analysis (PCoA) is depicted in two dimension using 100 markers for determining the genetic relatedness among the genotypes (Fig. 5). The first two components accounted for 36.45 and $6.16 \%$ of total inertia. The panel population containing 102 genotypes used in the study were distributed in four quadrants showing three major groups (Fig. 5). The 1st, 2nd, 3rd and 4th quadrant consisted of 24, 46, 24 and 8 number of genotypes, respectively. The genotypes belonging to three different sub-populations are grouped in different quadrants. The 2nd quadrant genotypes are divided into two groups of which one group is closer to axis 1 and another is to axis 2. These 2nd group genotypes closer to axis 2 are admix type depicted in red colour (Fig. 5a).

The majority of the genotypes containing moderate to high Fe and Zn content were placed in the 1st (top right) and 2nd (bottom right) quadrants of the PCoA. All the genotypes that are moderate to high for both zinc and iron namely, IET23834, Chittimuthyalu, IET24780, IET24771, IET24766, IET24783, IET24772, IET25443, IET25444, IET25447, IET25449, IET25450, IET25452, IET25453, IET25463, IET25465, IET25469, IET25471, IET25474, IET25479 and Safed Luchai-2 are placed in the red encircled area except five genotypes Kalanamak, IET24557, IET24787, IET25454 and IET25473 located in 1st quadrant (top right) and CR Dhan 907 in the 3rd quadrant (Fig. 5b). The genotypes namely Chittimuthyalu, IET25450, IET25450 and IET25465 containing high zinc and iron are placed in the red encircled area including Kalojeera, Kalonunia, Swarnakranti and IET25464. The green encircled area consisted of mostly the moderate grain Fe and/or Zn containing genotypes, whereas the blue encircled area covering the 3rd and 4th quadrant included mostly the germplasm lines low to Fe and/or Zn content (Fig. 5b).

The cluster analysis based on genotyping of panel population with the 100 markers was performed to determine their collective discrimination ability. The tree constructed with unweighted-neighbour joining method could differentiate the genotypes into 3 different clusters (Fig. 6). The cluster I, II and III consisted of 32, 51 and 19 genotypes, respectively. The three clusters clearly separated the three sub-populations obtained by STRUCT URE analysis. The sub-populations SP1, SP2 and SP3 were grouped separately under cluster III (green), II (blue) and I (pink), respectively (Fig. 6a). The admix genotypes, Kalonunia, Kalanamak, Kalojeera, Swarnakranti, IET25464 formed a distinct sub-cluster under cluster II remaining together with SP2. Significant correlation was observed between the iron and zinc content of rice grain and clustering pattern (Fig. 6b, c). The cluster depicted in red color indicate high, green for moderate and blue

LD plot

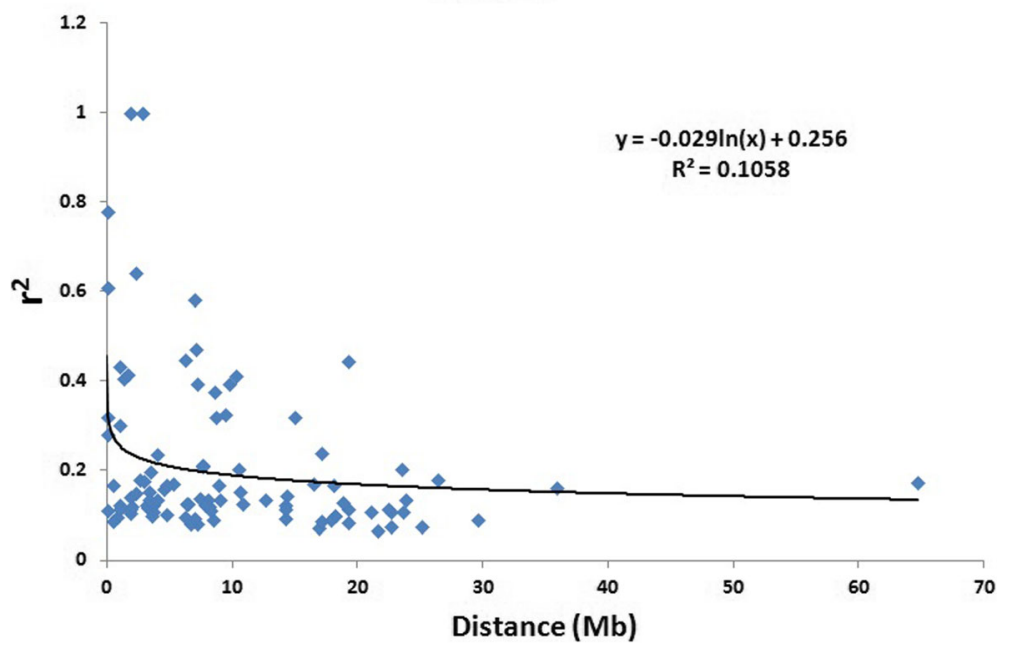

Fig. 4 Linkage disequilibrium (LD) decay $\left(r^{2}\right)$ curve plotted against the physical distance (base pairs, bp) between pairs of loci on chromosomes in rice. The decay started in million bp estimated by taking 95th percentile of the distribution of $r^{2}$ for all unlinked loci 


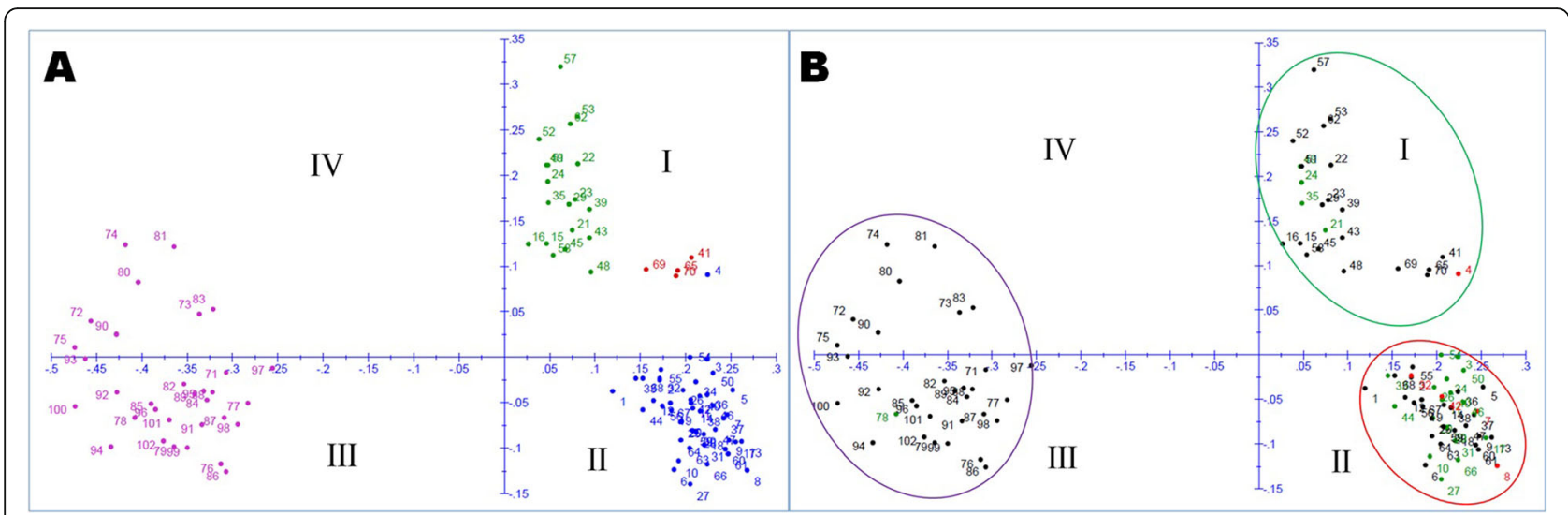

Fig. 5 Principal coordinate analysis (PCOA) of 102 genotypes in the panel population for grain Fe and Zn content using 100 molecular markers. The dot numbers in the figure represent the serial number of the genotypes enlisted in Table 1. The numbers are coloured on the basis of (a) sub-populations obtained from structure analysis (SP1-green; SP2-blue; SP3-Pink; admix type-red) (b) grain Fe and Zn content (common high grain Fe and Zn content - red; common moderate grain Fe and Zn content - green)

for low iron/zinc content in the tree. When iron content was compared, cluster I consisted 30 low iron and 2 (CR Dhan 907 and Nuadhusura) moderate in iron containing genotypes, Cluster III included majority of the high and moderate Fe containing genotypes. Of the 51 genotypes in the cluster II, more than $80 \%$ are low to moderate for grain $\mathrm{Fe}$ content. Hence, cluster II and III can be classified as moderate to high Fe containing group. Similarly, when $\mathrm{Zn}$ content of rice grain was compared with clustering pattern, cluster II and III included majority of the genotypes with high Zn (Fig. 6c). In cluster III, only three genotypes out of 19 genotypes were low for $\mathrm{Zn}$ content, where rest were moderate to high $\mathrm{Zn}$. Six genotypes with high and 28 genotypes with moderate $\mathrm{Zn}$ content were included in the cluster II of the tree (Fig. 6c).
Association of marker alleles with grain $\mathrm{Fe}$ and $\mathrm{Zn}$ content in rice

Association of grain $\mathrm{Fe}, \mathrm{Zn}$ content, panicles $/ \mathrm{m}^{2}$ and grain yield with the molecular markers was estimated using TASSEL 5 software. The association parameters were based on Generalized Linear Model (GLM) and Mixed Linear Model (MLM/ K + Q model). The marker-trait comparisons were subjected to filtration at less than $5 \%$ error i.e. $95 \%$ confidence ( $p$ value $<0.05$ ). The GLM approach computed average $\mathrm{r}^{2}$ value of 0.1133 with upper limit of 0.6095 and lower limit of 0.0386, whereas MLM showed $\mathrm{r}^{2}$ values ranging from 0.0392 to 0.146 with an average of 0.0622 (Additional file 5: Table S3; Additional file 6: Table S4). With $\mathrm{r}^{2}>0.10$ and $p \leq 0.05$ filter, 42 and 3 markers showed association with grain $\mathrm{Fe}$ and $\mathrm{Zn}$ content respectively by using GLM model (Additional file 5: Table S3). However, taking MLM model, only one marker GRMm9-1 showed

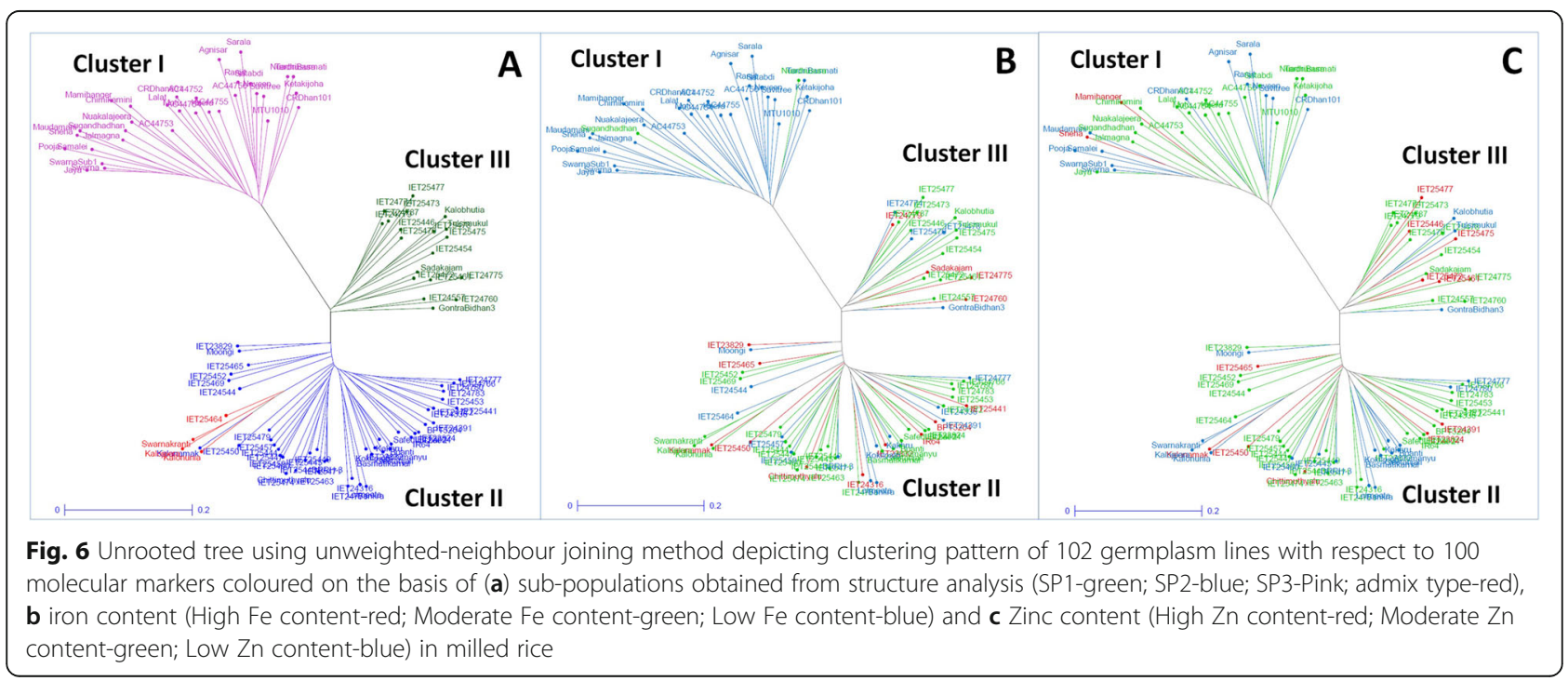


association with grain Zn content (Table 5). But when $\mathrm{r}^{2}>$ 0.05 was taken into account, 11 (RM243, RM122, RM234, RM80, RM315, RM339, RM1132, GRMM9-1, OsZIP8A, OsYSL2A, OsZIP6A) and 3 (RM80, RM300, RM1132) markers were associated with grain Fe and $\mathrm{Zn}$ content, respectively (Additional file 5: Table S3; Additional file 6: Table S4). Considering both the GLM and MLM, ten and seven markers showed significant association with grain $\mathrm{Fe}$ and $\mathrm{Zn}$ content, respectively at $p<0.05$ (Table 5). Four primers namely RM80, RM300, RM1132 and GRMM9-1

Table 5 Association of marker alleles with Fe and Zn content in milled rice detected both in GLM and MLM analyses in a shortlisted panel population of 102 genotypes

\begin{tabular}{|c|c|c|c|c|c|c|c|c|c|}
\hline \multirow[t]{2}{*}{ Trait } & \multirow[t]{2}{*}{ Marker } & \multicolumn{4}{|l|}{ GLM } & \multicolumn{4}{|l|}{ MLM } \\
\hline & & $\overline{F \text { value }}$ & $P$ value & $R^{2}$ & $q$ value & $\overline{F \text { value }}$ & $P$ value & $\mathrm{R}^{2}$ & $q$ value \\
\hline \multirow[t]{10}{*}{ Fe content } & RM243 & 6.21777 & 0.01429 & 0.05854 & 0.01958259 & 5.85605 & 0.01733 & 0.05798 & 0.03987069 \\
\hline & RM122 & 4.3147 & 0.04035 & 0.04136 & 0.04265571 & 7.11419 & 0.00892 & 0.07044 & 0.027565 \\
\hline & RM234 & 16.81381 & $8.41 \mathrm{E}-05$ & 0.14394 & 0.00062208 & 8.05498 & 0.0055 & 0.07975 & 0.027565 \\
\hline & RM7 & 8.87945 & 0.00362 & 0.08155 & 0.00558083 & 4.05058 & 0.04684 & 0.0401 & 0.04814111 \\
\hline & RM168 & 14.70112 & $2.20 \mathrm{E}-04$ & 0.12817 & 0.00093926 & 4.93231 & 0.02862 & 0.04883 & 0.03987069 \\
\hline & RM80 & 16.39877 & 1.01E-04 & 0.14088 & 0.0006253 & 7.60915 & 0.00691 & 0.07534 & 0.03987069 \\
\hline & RM339 & 11.69699 & $9.08 \mathrm{E}-04$ & 0.10472 & 0.00176731 & 5.24734 & 0.02408 & 0.05195 & 0.03987069 \\
\hline & RM1132 & 4.14297 & 0.04445 & 0.03978 & 0.04568472 & 5.61267 & 0.01975 & 0.05557 & 0.03987069 \\
\hline & OSZIP8 & 11.44893 & 0.00102 & 0.10273 & 0.001887 & 5.2546 & 0.02398 & 0.05203 & 0.03987069 \\
\hline & OSZIP6A & 5.86773 & 0.01722 & 0.05543 & 0.0205529 & 7.37666 & 0.00779 & 0.07304 & 0.027565 \\
\hline \multirow[t]{7}{*}{ Zn content } & RM260 & 5.99267 & 0.01611 & 0.05654 & 0.0205529 & 4.6087 & 0.03423 & 0.04563 & 0.04155935 \\
\hline & RM80 & 11.86398 & 8.38E-04 & 0.10606 & 0.00172177 & 5.28924 & 0.02354 & 0.05237 & 0.03987069 \\
\hline & RM300 & 11.98471 & 7.91E-04 & 0.10702 & 0.00172177 & 7.74249 & 0.00645 & 0.07666 & 0.027565 \\
\hline & RM339 & 5.32614 & 0.02307 & 0.05057 & 0.02586636 & 4.1887 & 0.04332 & 0.04147 & 0.04714235 \\
\hline & RM340 & 5.59171 & 0.01998 & 0.05296 & 0.02310188 & 4.78072 & 0.03111 & 0.04733 & 0.03987069 \\
\hline & RM1132 & 9.0313 & 0.00335 & 0.08283 & 0.00538913 & 6.3645 & 0.01322 & 0.06301 & 0.03762615 \\
\hline & GRMM9-1 & 13.00392 & 4.87E-04 & 0.11507 & 0.00137370 & 12.41426 & $6.44 \mathrm{E}-04$ & 0.12291 & 0.008732 \\
\hline \multirow[t]{12}{*}{ Panicles $/ \mathrm{m}^{2}$} & RM248 & 18.23846 & 4.45E-05 & 0.15425 & 0.00041122 & 5.4383 & 0.0217 & 0.05384 & 0.03987069 \\
\hline & RM17 & 14.62367 & $2.28 \mathrm{E}-04$ & 0.12758 & 0.00093926 & 11.26493 & 0.00112 & 0.11153 & 0.008732 \\
\hline & RM3392 & 9.9083 & 0.00217 & 0.09015 & 0.00364954 & 4.57817 & 0.03482 & 0.04533 & 0.04155935 \\
\hline & RM440 & 13.87657 & $3.23 \mathrm{E}-04$ & 0.12186 & 0.00119617 & 4.78325 & 0.03107 & 0.04736 & 0.03987069 \\
\hline & RM85 & 12.94277 & $5.01 \mathrm{E}-04$ & 0.1146 & 0.00137370 & 3.98538 & 0.04862 & 0.03946 & 0.04862 \\
\hline & RM421 & 7.28154 & 0.00818 & 0.06787 & 0.0121064 & 11.14884 & 0.00118 & 0.11038 & 0.008732 \\
\hline & RM31 & 4.76448 & 0.03139 & 0.04548 & 0.03415971 & 4.77291 & 0.03125 & 0.04726 & 0.03987069 \\
\hline & RM556 & 18.72712 & $3.58 \mathrm{E}-05$ & 0.15773 & 0.00041122 & 4.90142 & 0.02911 & 0.04853 & 0.03987069 \\
\hline & RM23 & 15.80571 & $1.33 \mathrm{E}-04$ & 0.13648 & 0.00070178 & 4.12242 & 0.04497 & 0.04082 & 0.04753971 \\
\hline & RM340 & 12.95157 & 4.99E-04 & 0.11466 & 0.00137370 & 5.74925 & 0.01835 & 0.05692 & 0.03987069 \\
\hline & OsNRAMP1A & 26.69468 & $1.22 \mathrm{E}-06$ & 0.2107 & $3.206975 \mathrm{e}-05$ & 11.87069 & 8.35E-04 & 0.11753 & 0.008732 \\
\hline & OsFER1 & 25.83769 & $1.73 \mathrm{E}-06$ & 0.20533 & 3.206975 e-05 & 9.85209 & 0.00223 & 0.09755 & 0.01375167 \\
\hline \multirow[t]{8}{*}{ Yield/plot } & RM243 & 4.05979 & 0.0466 & 0.03901 & 0.0466 & 4.3019 & 0.04064 & 0.04259 & 0.04556606 \\
\hline & RM248 & 5.92531 & 0.0167 & 0.05594 & 0.0205529 & 5.01002 & 0.02742 & 0.0496 & 0.03987069 \\
\hline & RM17 & 11.91911 & $8.16 \mathrm{E}-04$ & 0.1065 & 0.00172177 & 7.11104 & 0.00894 & 0.07041 & 0.027565 \\
\hline & RM517 & 12.86597 & $5.20 \mathrm{E}-04$ & 0.11399 & 0.00137370 & 14.7466 & $2.16 \mathrm{E}-04$ & 0.14601 & 0.027565 \\
\hline & RM421 & 6.89217 & 0.01002 & 0.06448 & 0.01425923 & 5.55647 & 0.02036 & 0.05501 & 0.03987069 \\
\hline & RM34 & 6.06991 & 0.01546 & 0.05723 & 0.02042929 & 5.16896 & 0.02513 & 0.05118 & 0.03987069 \\
\hline & RM339 & 11.30774 & 0.00109 & 0.10159 & 0.00192047 & 4.47416 & 0.0369 & 0.0443 & 0.04266563 \\
\hline & RM1132 & 11.952 & 8.03E-04 & 0.10676 & 0.00172177 & 4.78822 & 0.03098 & 0.04741 & 0.03987069 \\
\hline
\end{tabular}


and six primers viz. RM243, RM234, RM80, RM339, OsZIP8B, OsZIP6A were associated with grain $\mathrm{Zn}$ and $\mathrm{Fe}$ content, respectively with $\mathrm{r}^{2}>0.05$ and $\mathrm{p} \leq 0.05$ filter in both the models (Table 5). The Q-Q plot also confirmed the association of the markers with grain Fe and $\mathrm{Zn}$ content in rice (Fig. 7).

Agronomy traits like panicles $/ \mathrm{m}^{2}$ and grain yield also showed significant association with 44 and 25 primers, respectively using GLM at $P<0.05$ (Additional file 5: Table S3). The numbers reduced to 13 for each trait when analyzed with MLM model (Additional file 6: Table S4). However, considering GLM and MLM, twelve and eight markers showed significant association with panicles $/ \mathrm{m}^{2}$ and grain yield, respectively at $p<0.05$. Of these, nine markers (RM248, RM17, RM440, RM85, RM556, RM23, RM340, OsNARMP1A, OsFER1) with GLM and four markers (RM17, RM421, OsNARMP1A, OsFER1) with GLM as well as MLM showed $r^{2}>0.10$ for panicles $/ \mathrm{m}^{2}$. Similarly, for yield trait, RM17, RM517, RM339 and RM1132 were associated with $\mathrm{r}^{2}>0.10$ using GLM and that with both the models only RM517 showed $\mathrm{r}^{2}>0.10$ (Table 5). The Q-Q plot also confirmed the association of the markers with grain panicles $/ \mathrm{m} 2$ and grain yield in rice (Fig. 7).

Common markers were observed to be associated with different traits. Sixteen and four common markers were observed for Fe and Zn content using GLM and MLM model respectively (Additional file 5: Table S3 and Additional file 6: Table S4). This number reduced to three (RM80, RM339, RM1132) when both the models were considered simultaneously. Considering GLM model, 12 markers viz., RM243, RM488, RM248, RM17, RM3392, RM440, RM201, RM421, RM585, RM34, RM339 and RM1132 showed significant association with all the traits viz., grain $\mathrm{Fe}$ and $\mathrm{Zn}$ content, panicles $/ \mathrm{m} 2$ and yield. RM1132 and RM339 were commonly associated with grain Fe, $\mathrm{Zn}$ and yield considering both GLM and MLM models (Table 5). OsFER1 was associated with all the traits except grain yield.

\section{Quantitative real time- PCR for validation of associated markers/genes with grain Fe and $\mathrm{Zn}$ content}

The qRT-PCR analysis was performed for validating the identified markers in the marker-trait association analysis. Two significantly associated markers OsZIP8B and OsZIP6A for the genes OsZIP8 and OsZIP6 respectively were selected for validation study. The results of qRTPCR for the genes were normalized with housekeeping gene b-tubulin. The expression of the genes was studied in root as well as shoot of two genotypes Kalanmak (high grain $\mathrm{Fe}$ and $\mathrm{Zn}$ ) and Swarna (low grain $\mathrm{Fe}$ and $\mathrm{Zn})$ under $\mathrm{Fe}-\mathrm{Zn}$ normal and deficient condition. Both the genes were up-regulated under Fe-Zn deficient condition as compared to the control situation (Fig. 8). The fold change of expression of both the genes ranged 1.69-6.22 in deficient condition. Higher expression was obtained in root. Swarna showed more upreglation in roots as compared to Kalanamak.

\section{Discussion}

Modern high yielding varieties are poor in essential micronutrients like grain $\mathrm{Fe}$ and $\mathrm{Zn}$ content in rice $[16,17]$. On an average, polished rice has $2 \mathrm{mg} \mathrm{kg}^{-1}$ $\mathrm{Fe}$, while the recommended dietary intake for humans is $10-15 \mathrm{mg} \mathrm{kg}^{-1}$. Previous germplasm study for grain $\mathrm{Fe}-\mathrm{Zn}$ content reported variability range of 6.3-24.4 $\mathrm{mg} \mathrm{kg}^{-1}$ for $\mathrm{Fe}$ and $13.5-28.4 \mathrm{mg} \mathrm{kg}^{-1}$ for $\mathrm{Zn}$ in rice grain strongly suggests existence of genetic variability of these two micronutrients in rice germplasms $[6,8$, $9,25,52,53]$. Evaluation of the panel population containing 102 genotypes showed a maximum value of 4.48 $\mathrm{mg} \mathrm{kg}{ }^{-1} \mathrm{Fe}$ and $26.96 \mathrm{mg} \mathrm{kg}^{-1} \mathrm{Zn}$ content in the milled rice (Table 1). Further, biplot analysis revealed a wide variability in Fe and $\mathrm{Zn}$ content in the milled rice of the panel population (Fig. 2). It is observed that among the genotypes with $>4 \mathrm{mg} \mathrm{kg}^{-1} \mathrm{Fe}$ in milled rice showed $>12 \mathrm{mg}$ $\mathrm{kg}^{-1}$ in brown rice (Table 1). Similarly, the genotypes containing $>20 \mathrm{mg} \mathrm{kg}^{-1} \mathrm{Zn}$ in milled rice showed $>25 \mathrm{mg}$ $\mathrm{kg}^{-1}$ in brown rice. Therefore, mapping of QTLs

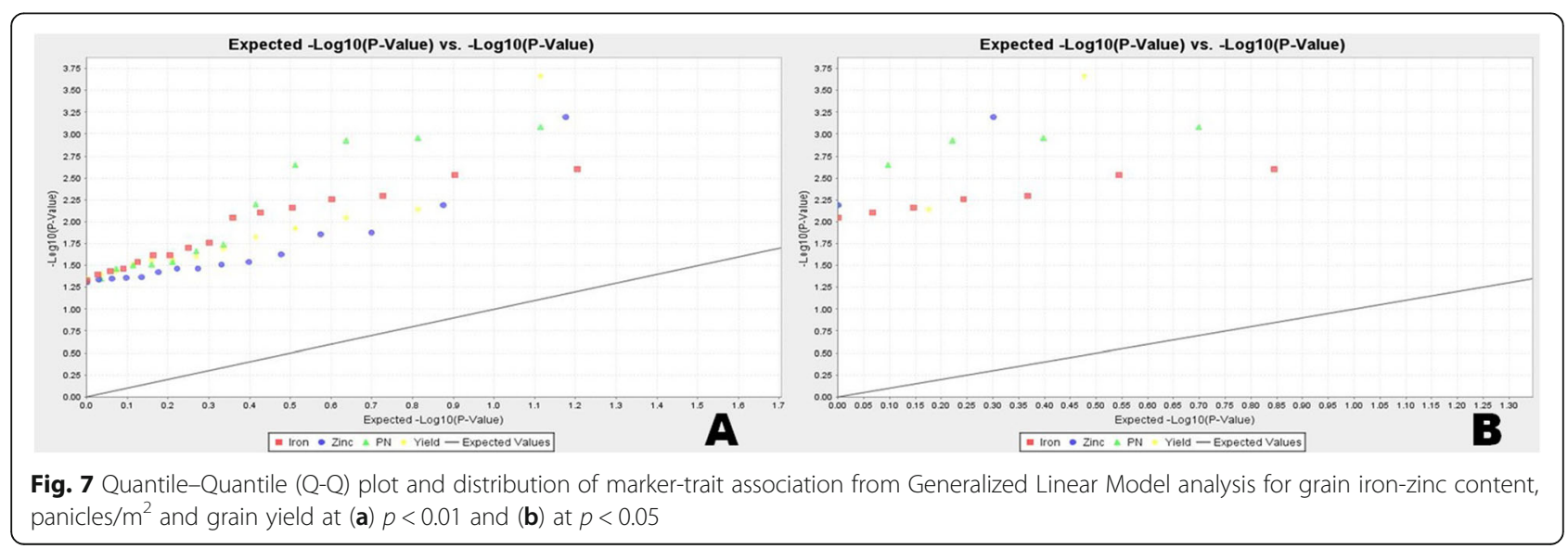




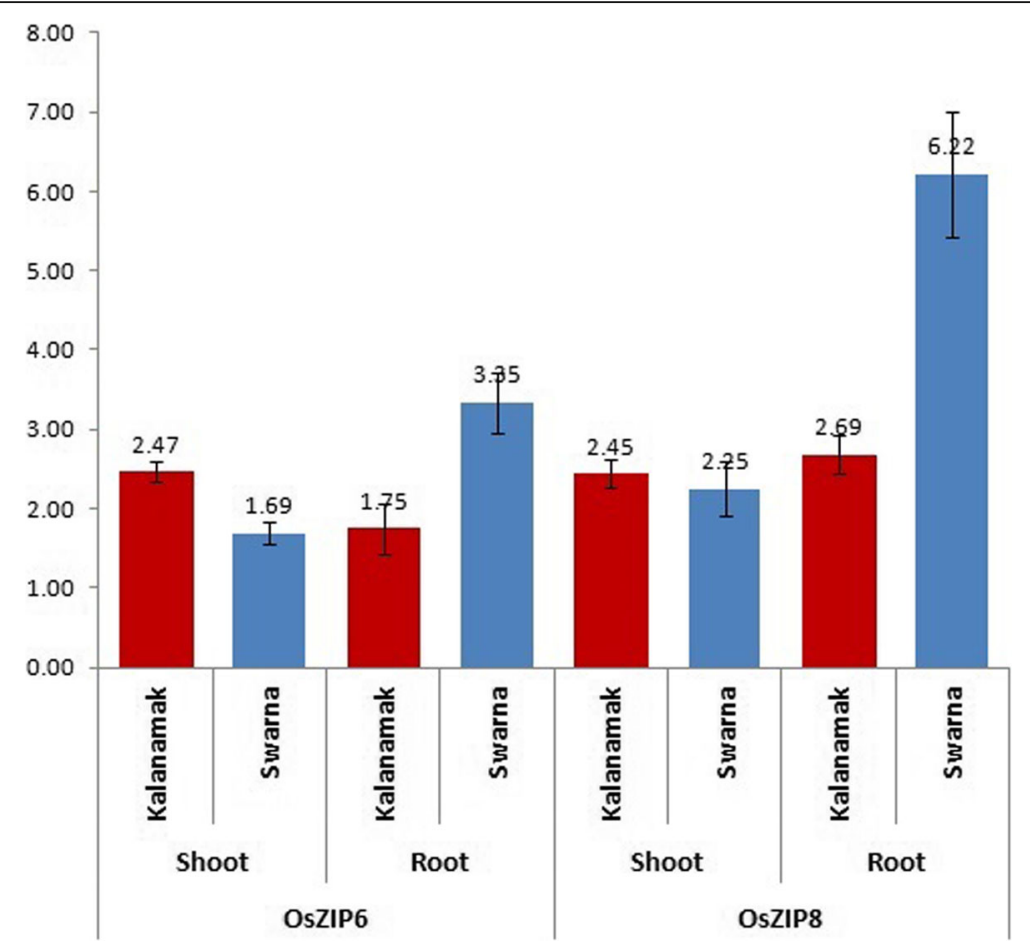

Fig. 8 Fold change of expression of genes OsZIP6 and OsZIP8 under Fe-Zn deficient treatment as compared to that of normal condition in roots and shoots of two contrasting genotypes Kalanamak and Swarna

responsible for high $\mathrm{Fe}$ and $\mathrm{Zn}$ content in the materials seems to be logical. Soil characteristics at the growing locations and genotype $\mathrm{x}$ environment has a great role in determining the grain micronutrient content in rice [17, 27]. Therefore, 2 years pooled data for phenotyping of grain $\mathrm{Fe}$ and $\mathrm{Zn}$ content is more reliable than single year. On the basis of 2 years mean data, the panel population was grouped into three classes based on their grain Fe and $\mathrm{Zn}$ content. Therefore, the population structure of the panel population for these traits is very important. Prichard's structure analysis at $\mathrm{K}=3$ also grouped the population into three distinct sub-populations.

Further, it was observed that a comparable grain Fe and $\mathrm{Zn}$ content were obtained in some varieties and biofortified lines as in the positive check varieties (Table 1). A positive correlation of $\mathrm{Fe}$ and $\mathrm{Zn}$ content in rice grain in some genotypes had been reported in previous publications [54-56]. Mean performance table generated here also revealed that few genotypes with high $\mathrm{Zn}$ content possessed high Fe content also (Table 1). Besides, evaluation results showed that some genotypes were with at par yield with the yield check varieties and contain moderate to high $\mathrm{Fe}-\mathrm{Zn}$ content in the grains. Therefore, improvement of $\mathrm{Fe}$ and $\mathrm{Zn}$ content in rice grain along with high grain yield is possible. Relationship of high grain yield with $\mathrm{Zn}$ content is reported in earlier publication [57]. A similar observation for grain protein content has been reported recently in rice [17]. From panel's genotype-trait biplot analysis, it is clear that $\mathrm{I}^{\text {st }}$ and 2 nd quadrant accommodated genotypes with better in $\mathrm{Fe}$ and $\mathrm{Zn}$ content along with better grain yield (Fig. 2). Previous mapping results also reported linkage of primer RM490 for Zn content associated with yield component QTLs like panicles/plant and 1000-grain weight [58]. Current association mapping revealed association of RM260 with Zn content in rice grain. Also, qDTY12.1 an-yield QTL under drought was reported to be linked to primer RM260 [36]. The same is associated with $\mathrm{Zn}$ content in rice grain (Table 5). Therefore, improvement of Fe- $\mathrm{Zn}$ content along with high grain yield is challenging but possible.

The principal component and coordinate analyses distributed the panel' population according to their grain Fe- $\mathrm{Zn}$ content along with grain yield and allocated distinct spots in the four quadrants (Figs. 2 and 5). Distribution of genotypes into different quadrants indicated the presence of genetic variation for Fe and $\mathrm{Zn}$ content in the panel population. The neighbour joining tree also grouped the germplasms into different classes on the basis of genotyping results of 100 molecular markers (Fig. 6). The figure showed various groups and sub-groups in the panel population based on genotyping using 100 markers for these traits clearly categorized the panel population into different groups indicating involvement of different genes/QTLs for these classes (Fig. 6). Presence of these different groups in the population increased the continuation of linkage 
disequilibrium and favoured for getting the marker-trait association for Fe-Zn content in rice grain. Similar results of trait mapping through marker-phenotype association were reported earlier for various rice phenotypic traits [17, 39, 40, 43, 45, 47]. A moderate level of genetic diversity was observed in the studied population for grain Fe and $\mathrm{Zn}$ content. The moderate genetic diversity detected in this study for Fe and $\mathrm{Zn}$ content is similar to the earlier results on genetic diversity obtained by other workers for these traits [53, 59-61]. However, rich genetic diversity values for these traits were also reported earlier in rice [6, 7, 9, 16, 62-64]. In our structure analysis, we observed a lower value of alpha $(\alpha=0.0388)$ indicating that these two traits had a common primary ancestor. Subsequently, it evolved with each sub-population with few admix genotypes. The values obtained from inferred ancestry indicated small effects of individual QTLs responsible for trait groups and subgroups in the population. These small effects QTLs need to be put together into a favourable high yielding background through molecular breeding approaches. Similar observations are available from earlier association studies also support this suggestions for gene(s) / QTLs stacking in popular varieties [17, 39, 40, 47].

The $\mathrm{F}_{\mathrm{ST}}$ values estimated for the pair of subpopulations showed a clear variation in the values. The $\mathrm{F}_{\mathrm{ST}}$ value for SP1 and SP2 was found to be moderate $(0.247)$ value. The variation in $\mathrm{F}_{\mathrm{ST}}$ values and their pattern of distribution revealed a clear cut differentiation among the sub-populations from each other with respect to $\mathrm{Fe}$ and $\mathrm{Zn}$ content in the grains. Thus, the population is categorized into clear genetic groups based on the inter and intra-sub $\mathrm{F}_{\mathrm{ST}}$ values. It is therefore expected that the populations and individuals showing higher $\mathrm{F}_{\mathrm{ST}}$ values may produce better progenies containing $\mathrm{Fe}$ and $\mathrm{Zn}$ content in recombination breeding. Therefore, attempt to pyramid these QTLs responsible for higher Fe and $\mathrm{Zn}$ may be put from different populations lead to higher $\mathrm{Fe}$ and $\mathrm{Zn}$ content in the progenies. Similar suggestions were also provided by earlier workers for increasing high and low temperature stress tolerance and grain yield in rice [17, 39, 40,65]. A sharp decline and then very slow and gradual LD decay value was noticed in the panel population (Fig. 4). This may be due to the self pollination nature of rice crop. The LD decay is usually high in open pollinated crops.

TASSEL analysis detected marker and grain $\mathrm{Fe}-\mathrm{Zn}$ content association in the tested panel population. Higher $\mathrm{F}$ and lower $p$ value with high $r^{2}$ were detected for 10 and 7 markers associated with Fe and $\mathrm{Zn}$ content in kernel, respectively. These were the reliable markers to be used in $\mathrm{Fe}$ and $\mathrm{Zn}$ enhancement breeding program as detected through both GLM and MLM analyses. Thus, suggesting a strong association of marker with grain $\mathrm{Fe}-\mathrm{Zn}$ content in the panel population. This is further evident from Q-Q plot exhibiting expression of many QTLs for grain Fe and Zn content (Fig. 7). This marker-trait association provides clue for presence of various QTLs in the germplasms containing more grain Fe and $\mathrm{Zn}$. It may be due to the involvement of different donor lines carrying different QTLs for these two traits. The accumulation of useful QTLs for these traits in few landraces may be possible by natural introgression over a long time period ultimately exhibiting a higher quantity of the two micronutrients in those germplasm lines. Similar predictions for accumulation of useful QTLs in stress tolerant wild and landraces genotypes were also earlier reported in rice $[17,39,40]$.

All the significantly associated markers for grain Fe content namely RM243, RM80, RM339, OsZIP6A and OsZIP8B detected through both GLM and MLM models with $\mathrm{r}^{2}>0.05$ and high $\mathrm{F}$ value indicated a very strong association. Probably, these QTLs contribute to variability for Fe content in rice grain. Some of these markers were reported to be linked to Fe enhancing QTLs in rice. Similarly, four markers namely RM80, RM300, RM1132 and gRMm9-1 showed significant association with $\mathrm{Zn}$ content detected through both the models at $r^{2}>0.05$. These primers are located in different chromosomes which may correspond to different QTLs (Table 5).

Ten markers namely RM243, RM122, RM234, RM7, RM168, RM80, RM339, RM1132, OsZIP6A and OsZIP8B showed significant association with grain iron content detected with both GLM and MLM models. RM243, RM168, RM122, RM234 and RM80 are earlier reported for qFe1.1, qFe3.1, qFe5.1, qFe7.1 and qFe8.1 QTLs, respectively [9]. The marker RM339 is physically located closer to RM80 (qFe8.1), it will be useful in marker-assisted transfer of qFe8.1. Hence, these six markers and five QTLs were validated using the panel population and these reliably can be deployed in MAS breeding for improvement of Fe content in rice grain. OsZIP8 and OsZIP6 are Zinc-iron transporters belonging to ZIP family of micronutrient transporters. Particularly, OsZIP8 is important for Zn transport to seed [66]. Both these transporters being associated with grain $\mathrm{Fe}$ and $\mathrm{Zn}$ content in our study imply that both of these genes are also responsible for Fe transport to seed. These two genes were also reported earlier and currently detected by both the models $[8,28,66]$. Therefore, these two markers can reliably be used for $\mathrm{Fe}$ enhancement. Two markers RM7 and RM1132 were reported earlier for $q Z n 3.1$ and $q Z n 7$, respectively for grain $\mathrm{Zn}$ content $[9,67]$. However, these two primers were now strongly associated for grain Fe content detected by both the models. QTLs controlling Fe content located in these locations of the two chromosomes were not reported earlier. Hence, these two locations can be designated as 
QTLs $q F e 3.3$ and $q F e 7.3$ and are novel QTLs. Two primers, RM340 and RM1132 reported for $q Z n 6$ and $q Z n 7$ are now detected to be strongly associated with grain Zn content [67]. Hence, these two QTLs are validated and reliably may be deployed for $\mathrm{Zn}$ enhancement in rice grain. Two markers namely RM339 and gRMm9-1 (LOC_Os09g27330: oxidoreductase/ transition metal binding protein) were reported earlier and currently detected by both the models $[8,15]$. Therefore, these two markers can also reliably be used for $\mathrm{Zn}$ enhancement. Two primers RM80 and RM260 were reported earlier for $q F e 8.1$ and $q F e 12.2$ for grain $\mathrm{Fe}$ content [9]. However, these two primers under this study were strongly associated for grain $\mathrm{Zn}$ content detected by both the models. QTLs controlling Zn content present in these regions of the chromosomes were not reported in previous publications. Hence, these two positions on chromosome 8 and 12 are designated as QTLs $q Z n 8.3$ and $q Z n 12.3$ and considered to be novel QTLs controlling grain $\mathrm{Zn}$ content in rice. RM300, located on chromosome 2 , is detected to be strongly associated with $\mathrm{Zn}$ content. No earlier reports could be found, hence, designated as $q Z n 2.2$ that controls grain $\mathrm{Zn}$ content. From mapping study, it is reported that RM1132 is associated with grain $\mathrm{Zn}$ content with $q Z n 7$ controlling the trait [67]. However, we detected a strong association for grain Fe content in the same location on the chromosome 7. As these locations on chromosome 7, 8 and 12 are commonly responsible for $\mathrm{Zn}$ and Fe content, is can be said that these Fe and Zn enhancing QTLs may be co-localized.

Several markers strongly associated with grain Fe and/ or $\mathrm{Zn}$ content were observed with single model (GLM or MLM). The different markers used for zinc/iron transporters belonging to ZIP family, yellow stripe gene family (YSL), ferritin, metal tolerance protein (MTP) family, Natural Resistance Associated Macrophage protein 1 (NRAMP) family were associated with Fe content indicating common transport mechanism for Fe and $\mathrm{Zn}$. Ferritins are iron storage proteins having two functional units for rapid uptake of $\mathrm{Fe}^{2+}$ and nucleation of $\mathrm{Fe}^{3+}[68$, 69]. Association of OsFER1 with $\mathrm{Zn}$ content and panicle number may imply the involvement of this gene with $\mathrm{Zn}$ storage along with Fe thereby increasing panicle number of plants as both are essential micronutrients for plant growth. As plant ferritins lack iron response elements [69]. There may be chance of carrying $\mathrm{Zn}$ along with $\mathrm{Fe}$. But this needs further confirmation with detailed experimentation.

Markers associated with $\mathrm{Fe}$ and $\mathrm{Zn}$ were analysed to see their involvement in enhancement of yield and its component traits. Twelve markers viz., RM243, RM488, RM248, RM17, RM3392, RM440, RM201, RM421, RM585, RM34, RM339, RM1132 showed significant association with grain
Fe and $\mathrm{Zn}$ content as well as panicles $/ \mathrm{m}^{2}$ and yield considering GLM model only (Additional file 5: Table S3). This indicated that the QTLs controlling these four traits may be co-localized and hence higher chance of coinheritance. Similarly two markers RM1132 and RM339 were commonly associated with grain $\mathrm{Fe}, \mathrm{Zn}$ and yield detected considering both GLM and MLM (Table 5). The QTLs controlling grain weight, qTBGW8 [70] and plant architecture, OsSPL14 [71] are located only $0.46 \mathrm{Mb}$ and $7.33 \mathrm{Mb}$ apart, respectively, from RM339 located on chromosome 8. Also, RM1132 is located only $5.1 \mathrm{Mb}$ away from $q P N 7$ reported to control panicle number of rice plant [70]. These observations provide clues for common inheritance of these QTLs. Hence, enhancement of both the traits viz., grain $\mathrm{Fe}$ and $\mathrm{Zn}$ content and yield parameters can easily be achieved simultaneously. Also, in a recent publication, it is established that grain Fe- $\mathrm{Zn}$ can easily be improved with few yield component traits [36]. Similar was the case of grain protein content heritability, negative relationship with grain yield and influence of genotype and environment interaction [18-21]. However, the recent results published on protein content mapping in rice indicated about the possibility of improving grain protein content along with high grain yield [17].

The qRT-PCR analysis was performed for validating the identified markers in the marker-trait association analysis. The expression of the two studied genes OsZIP8 (Fe-Zn transporter) and OsZIP6 ( $\mathrm{Zn}$ transporter) under Fe-Zn normal condition and their upregulation in deficient condition confirmed the accuracy of the association study. Hence, it can be concluded that other QTLs significantly associated will be useful. Both OsZIP8 and OsZIP6 were up-regulated under Fe-Zn deficient condition as compared to the control situation (Fig. 8). Earlier studies also reported higher expression of these genes under deficient condition [72, 73]. Higher expression of both the genes in roots of Swarna (low grain $\mathrm{Fe}$ and $\mathrm{Zn}$ ) under deficient condition indicates more uptake of the nutrient in the roots but it may not be getting properly channelized to the grain as evident from lower upregulation in shoots of Swarna, It might be utilising the nutrient more for its survival rather storing in the grains. But in case of Kalanamak (high grain Fe and $\mathrm{Zn}$ ), both the genes showed almost equal upregulation in roots and shoots thereby translocating $\mathrm{Zn}$ and $\mathrm{Fe}$ to the grain.

\section{Conclusion}

Polished rice consumed in India is very low in iron and zinc micronutrient content. Phenotyping results categorized genotypes based on grain $\mathrm{Fe}$ and $\mathrm{Zn}$ content each into 3 sub-classes. Evaluation results indicated that 3 genotypes namely IET24779, IET25465 and Chittimuthyalu produced at par yield with standard check 
variety IR64 along with high grain Fe-Zn content. Therefore, it is possible to improve grain $\mathrm{Fe}$ and $\mathrm{Zn}$ content in rice along with high grain yield. However, the studied panel showed a moderate level of genetic diversity for $\mathrm{Fe}$ and $\mathrm{Zn}$ content based on 100 molecular markers. Linkage disequilibrium for $\mathrm{Fe}$ and $\mathrm{Zn}$ content was observed in the studied population showing deviation from Hardy-Weinberg's expectation based on F statistic values. The analysis detected $38 \%$ of the variation among populations, $52 \%$ among individuals and $10 \%$ within individuals in the studied panel population. The panel population was categorized into three sub-populations by the STRUCTURE analysis. The analysis also revealed a common primary ancestor for each sub-population with few admix individuals. Association mapping detected 10 QTLs for grain $\mathrm{Fe}$ and 7 for grain $\mathrm{Zn}$ content to be strongly associated through both Generalized Linear Model (GLM) and Mixed Linear Model (MLM). Novel QTLs namely $q F e 3.3$ and $q F e 7.3$ for grain Fe and $q Z n 8.3$ and $q Z n 12.3$ for grain $Z n$ content were detected. Four QTLs controlling grain $\mathrm{Fe}$ and $\mathrm{Zn}$ were detected to be co-localized on the same chromosome. Besides, some Fe-Zn controlling QTLs were detected to be co-localised with the yield and its component QTLs. Incorporation of these QTLs may enhance grain yield too. The strongly associated markers with QTLs controlling high grain $\mathrm{Fe}-$ $\mathrm{Zn}$ namely qFe1.1, qFe3.1, qFe5.1, qFe7.1, qFe8.1, OsZIP8B (Zn-Fe regulated transporter), OsZIP6A (LOC_ Os05g0164800), qFe3.3 and qFe7.3 for $\mathrm{Fe}$ and qZn6, $q Z n 7, q Z n 2.2, \quad g R M m 9-1$ (LOC_Os09g27330), qZn8.3 and $q Z n 12.3$ will be useful QTLs in stacking for developing nutrient dense rice.

\section{Methods}

\section{Plant materials and field experiment}

A total of 485 germplasm lines were screened for Fe and Zn content of milled rice during 2013 to 2015 [74-76]. The landraces and cultivars were obtained from ICARNational Rice Research Institute (NRRI), gene bank while the Fe- $\mathrm{Zn}$ biofortified lines were from national coordinated trials. A panel population containing 102 shortlisted genotypes representing the three distinct phenotypic classes for grain Fe-Zn content and three check varieties were included in the panel for association study of the micronutrient contents in rice (Additional file 3: Table S1). The cultivars, Kalanamak and Chittimuthyalu for high Fe-Zn content while IR64 for grain yield were used as checks. The genotypes were planted in randomized block design with three replications during wet season, 2016 and 2017 at ICAR-NRRI, Cuttack following a standard procedure for a good crop. Phenotypic performances of the genotypes for days to $50 \%$ flowering, panicles $/ \mathrm{m}^{2}$, iron and zinc content in milled rice and grain yield were recorded during both the years.
Data analyses of the traits were performed using the software CROPSTAT v7.0.

\section{Phenotyping for grain iron and zinc content in rice}

Each harvested paddy sample was processed to white rice using Krishi international $\mathrm{H}-810$ non-ferrous Huller and Krishi international K-710 polisher. Before dehusking, the grains were washed with $0.1 \mathrm{~N} \mathrm{HCl}$ followed by rinsing with double distilled water to make the sample free from contamination. Iron and zinc content $(\mathrm{mg} / \mathrm{Kg})$ were determined at ICAR-Indian Institute of Rice Research, Hyderabad using energy dispersive $\mathrm{x}$-ray fluorescence spectrophotometer (ED-XRF) X-supreme 8000 from $5 \mathrm{~g}$ polished rice samples. The published protocol of ED-XRF was used to estimate both the micro-nutrients [11]. These genotypes were grouped into three different phenotypic classes based on milled rice grain iron content for our study as high $\left(>4 \mathrm{mg} \mathrm{kg}^{-1}\right)$, medium (3-4 $\mathrm{mg} \mathrm{kg}^{-1}$ ) and low $\left(<3 \mathrm{mg} \mathrm{kg}^{-1}\right)$ and for zinc content as high $(>20 \mathrm{mg}$ $\left.\mathrm{kg}^{-1}\right)$, medium (15-20 $\left.\mathrm{mg} \mathrm{kg}^{-1}\right)$ and low $\left(<15 \mathrm{mg} \mathrm{kg}^{-1}\right)$. Principal component analysis (PCA) and scatter plots were generated as per standard procedure following previous publications [39, 40,77].

\section{DNA isolation, PCR amplification and visualization of markers for $\mathrm{Fe}$ and $\mathrm{Zn}$ content in polished rice}

The seedlings of the 102 genotypes were grown and the leaf samples were collected from 20 days old seedling for genomic DNA isolation. The DNA was isolated following standard protocol [78]. One hundred SSR and gene specific markers across the genome were used for the study (Additional file 4: Table S2). The gene specific markers were selected based on reported literature on $\mathrm{Fe}-\mathrm{Zn}$ content in rice. PCR was performed in $20 \mu \mathrm{l}$ reaction volume and amplified by following our earlier report [17]. Agarose gel (3.5\%) in TBE buffer ( $\mathrm{pH}$ 8.0) was used for electrophoresis. Ethidium Bromide stain was used for visualisation of the amplicons. $50 \mathrm{bp}$ DNA ladder was used to determine the size of the amplicon.

\section{Analyses for determination of population structure, genetic diversity parameters and association of markers} A matrix was constructed on the basis of presence and/or absence of alleles for each genotype-primer combination. PowerMarker Ver3.25 software was used for estimating allele number, polymorphic information content (PIC), allele frequency, gene diversity and heterozygosis [79]. NEI coefficient for dissimilarity index was calculated and unweighted neighbor joining un-rooted tree was constructed [80] with bootstrap value of 1000 by using DARwin 5 program [81]. A Bayesian clustering approach was followed for estimation of genetic structure by taking probable sub- 
populations (K) and higher delta K-value using the STRUCTURE 2.3.6 software [82]. A 150,000 burn-in period with 150,000 MCMC replications and $\mathrm{K}$ value run of 10 times was used for estimating population structure. The highest value of delta $\mathrm{K}$ estimated from Evanno table was taken as number of probable sub-populations present in the model and used in next step to detect the subpopulation values in the panel population $[17,39,40,83$, 84]. The deviation from Hardy-Weinberg expectation within and between the population structures $\left(\mathrm{F}_{\mathrm{IT}}, \mathrm{F}_{\mathrm{IS}}\right.$, $\mathrm{F}_{\mathrm{ST}}$ ) was estimated through Analysis of molecular variance (AMOVA) using GenAlEx 6.5 software [85]. Marker-trait association for $\mathrm{Fe}$ and $\mathrm{Zn}$ content of polished rice was performed using TASSEL 5 program [86].

Linkage disequilibrium plot was constructed following earlier reports [17]. In order to enhance accuracy of marker- trait association, false discovery rate (FDR) and adjusted $p$ values ( $q$ values) were calculated using the methods described in previous studies [40].

\section{Gene expression analysis by quantitative real time-PCR}

Two genotypes Kalanmak (high grain $\mathrm{Fe}$ and $\mathrm{Zn}$ ) and Swarna (low grain $\mathrm{Fe}$ and $\mathrm{Zn}$ ) were used for studying expression level of two genes OsZIP6 and OsZIP8 under $\mathrm{Fe}-\mathrm{Zn}$ normal and deficient condition. Eleven day old seedlings were exposed to Fe- $\mathrm{Zn}$ normal and deficient condition under hydroponics culture in Yoshida growth medium was used for control, whereas both Fe and $\mathrm{Zn}$ were omitted from Yoshida medium for deficiency condition [72, 73, 87]. Expression analysis in response to FeZn deficiency was performed for OsZIP6 and OsZIP8 genes using real-time primers [72]. Prior to real-time expression analysis, the total mRNA isolation, yield and purity evaluation of RNA were performed following the methods of earlier reports [88]. The increase/decrease of expression (fold change) of the OsZIP6 and OsZIP8 genes under Fe- $\mathrm{Zn}$ deficiency condition as compared to controlled condition was calculated by using $2^{-\Delta \Delta} \mathrm{Ct}$ method [89], where normalization was performed with rice $\beta$-tubulin gene [88]. Three technical replicates each from three independent biological replicates were taken used for the study following earlier reports [88].

\section{Supplementary information}

Supplementary information accompanies this paper at https://doi.org/10. $1186 /$ s12870-020-2262-4.

Additional file 1: Figure S1. Scree plot generated showing four component traits and eigen values generated by using 102 rice genotypes.

Additional file 2: Figure S2. The distribution pattern of $F_{S T}$ values (A) in the two sub-populations at $K=2(B)$ three sub-populations at $K=3$ showing a symmetric shape.

Additional file 3: Table S1. Days to $50 \%$ flowering, grain Fe content, Zn content, panicles $/ \mathrm{m}^{2}$ and grain yield of 102 genotypes including biofortified lines and check varieties studied during wet season, 2016 and 2017.

Additional file 4: Table S2. Information on the selected 100 molecular markers used for $\mathrm{Zn}$ and Fe content in indica rice.

Additional file 5: Table S3. Association of marker alleles with Fe, Zn content, panicle number and grain yield/plot in rice detected both in GLM analyses in a shortlisted panel population of 102 genotypes.

Additional file 6: Table S4. Association of marker alleles with Fe, Zn content, panicle number and grain yield/plot in rice detected both in MLM analyses in a shortlisted panel population of 102 genotypes.

\section{Abbreviations}

AMOVA: Analysis of molecular variance; CTAB: Cetyl Trimethyl Ammonium Bromide; FDR: False discovery rate; $F_{I S}$ : Fixation index within a population;

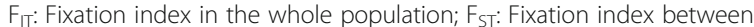
subpopulations; GLM: Generalized Linear Model; LD: Linkage disequilibrium; MCMC: Markov Chain Monte Carlo; MLM: Mixed Linear Model; PCA: Principal component analysis; PIC: Polymorphic information content; QTL: Quantitative trait loci

Acknowledgements

We thank Director, ICAR-NRRI, Cuttack for providing all facilities for the experiment.

Authors' contributions

SKP and EP conceived and designed the experiments. EP SKP SP DKN SRB RN SPM SKG and SRD performed the experiments. EP SSCP analyzed the data. SKP JNR contributed reagents/materials/analysis tools. SKP EP wrote the paper. JNR edited the manuscript. All authors read and approved the final manuscript.

\section{Funding}

The work was financially supported as part of the Institute project of ICARNational Rice Research Institute, Cuttack, Odisha, India, under Indian Council of Agricultural Research, New Delhi is duly acknowledged. Funding body has no role in the design of the study and collection, analysis, and interpretation of data and in writing the manuscript.

Availability of data and materials

All data generated or analysed during this study are included in this article.

Ethics approval and consent to participate

Not applicable.

Consent for publication

Not applicable.

Competing interests

The authors declare that they have no competing interests.

Author details

${ }^{1}$ ICAR-National Rice Research Institute, Cuttack, Odisha, India. ${ }^{2}$ ICAR-Indian Institute of Rice Research, Hyderabad, India.

Received: 17 January 2019 Accepted: 20 January 2020

Published online: 04 February 2020

References

1. Shi Z, El-Obeid T, Li M, Xu X, Liu J. Iron-related dietary pattern increases the risk of poor cognition. Nutr J. 2019;18:48. https://doi.org/10.1186/s12937019-0476-9.

2. Allen $\mathrm{LH}$, De Benoist $B$, Dary $\mathrm{O}$, Hurrell R, editors. Guidelines on food fortification with micronutrients, vol. 57-61. Geneva: World Health Organization; 2006. p. 124-5.

3. Qui LC, Pan J, Dan BW. The mineral nutrient component and characteristic of color and white brown rice. Chinese J Rice Sci. 1995;7(2):95-100.

4. Ahmed SA, Borua I, Das D. Chemical composition of scented rice. Oryza. 1998;35(2):167-9. 
5. Graham R, Senadhira D, Beebe S, Iglesias C, Monasterio I. Breeding for micronutrient density in edible portions of staple food crops: conventiona approaches. Field Crops Res. 1999;60:57-80.

6. Gregorio GB, Senadhira D, Htut H, Graham RD. Breeding for trace mineral density in rice. Food Nutr Bull. 2000;21(4):382-6.

7. Zhang ZH, Su L, Chen W, Li W, Zhu YG. A major QTL conferring cold tolerance at early seedling stage using recombinant inbred lines of rice (Oryza sativa L.). Plant Sci. 2005;168:527-34.

8. Brar B, Jain S, Singh R, Jain RK. Genetic diversity for iron and zinc contents in a collection of 220 rice (Oryza sativa L.) genotypes. Indian J Genet. 2011; 71:67-73.

9. Anuradha K, Agarwal S, Batchu AK, Babu AP, BPM S, Longvah T, Sarla N. Evaluating rice germplasm for iron and zinc concentration in brown rice and seed dimensions. J Phytol. 2012:4(1):19-25.

10. Roja V, Kiranmayi SL, Sarla N. Enrichment of iron and zinc concentration in introgression lines of brown rice. Trends Biosciences. 2013;6:870-5.

11. Rao SP, Madhubabu P, Kota S, Bhadana VP, Varaprasad GS, Surekha K, Neerja $\mathrm{CN}$, Babu VR. Assessment of iron and zinc variability in rice germplasm using energy dispersive $x$-ray florescence spectrophotometer (ED-XRF). J Rice Res. 2014;7:45-52.

12. Patil R, Diwan JR, Nidagundi JM, Lokesha R, Ravi MV, Boranayak MB, Disshith S. Genetic diversity of brown rice for iron and zinc content. Electron J Plant Breed. 2015;6:196-203.

13. Swamy MBP, Rahman MA, Inabangan-Asilo M, Amparado A, Manito C, Mohanty C, Reinke R, Slamet-Loedin $1 \mathrm{H}$. Advances in breeding for high grain Zn in rice. Rice. 2016;9:49-57.

14. Zhang X, et al. Q+TL mapping for zinc content in rice grains. Chin Bull Bot. 2009;44:594-600.

15. Chandel G, Samuel P, Dubey M, Meena R. In silico expression analysis of QTL specific candidate genes for grain micronutrient ( $\mathrm{Fe} / \mathrm{Zn}$ ) content using ESTs and MPSS signature analysis in rice (Oryza sativa L.). J Plant Genet Trans. 2011;2:11-22.

16. Mahender A, Anandan A, Pradhan SK, Pandit E. Rice grain nutritional traits and their enhancement using relevant genes and QTLs through advanced approaches. Springplus. 2016;5(1):2086. https://doi.org/10.1186/s40064-0163744-6.

17. Pradhan SK, Pandit E, Pawar S, Bharati B, Chatopadhyay K, Singh S, Dash P, Reddy JN. Association mapping reveals multiple QTLs for grain protein content in rice useful for biofortification. Mol Gen Genomics. 2019;294:96383. https://doi.org/10.1007/s00438-019-01556-w.

18. Carnahan HL, Wiser WJ, Rutger JN. Associations between protein content and other characters in rice (abstr.). Proc. 14th Rice Tech. California: Working Group, Univ; 1972. p. 31-2.

19. HiileRisLambers D, Rutger JN, Qualset CO, Wiser WJ. Heritability of protein content and its relationship to agronomic characters in rice. In: Proc. 14th rice tech. California: Working group, Univ; 1972. p. 31.

20. Coffman WR, Juliano BO. Rice. In: Olson RA, Frey KJ, editors. Nutritional quality of cereal grains: genetic and agronomic improvement. Agronomy monograph no. 28. Madison: American Society of Agronomy; 1987. p. 101-31.

21. Yu YH, Li G, Fan YY, Zhang KQ, Min J, Zhu ZW, Zhuang JY. Genetic relationship between grain yield and the contents of protein and fat in a recombinant inbred population of rice. J Cereal Sci. 2009;50(1):121-5.

22. Biradar H, Vargavi MV, Sasalwad R, Parama R, Hittalmani S. Identification of QTLS associated with silicon and zinc content in rice and their role in blast resistance. Indian J Genet. 2007:67:105-9.

23. Stangoulis JCR, Huynh BL, Welch RM, Choi EY, Graham RD. Quantitative trait loci for phytate in rice grain and their relationship with grain micronutrient content. Euphytica. 2007;154:289-94.

24. Yongmei G, Ping M, Jiafu L, Yixuan L, Zichao L. QTL mapping and Q x E interactions of grain cooking and nutrient qualities in rice under upland and lowland environments. Acta Genet Sin. 2007;34:420-8.

25. Garcia-Oliveira AL, Tan L, Fu Y, Sun C. Genetic identification of quantitative trait loci for contents of mineral nutrients in rice grain. J Integr Plant Biol. 2009;51:84-92. https://doi.org/10.1111/j.1744-7909.2008.00730.x.

26. Lu K, Li L, Zheng X, Zhang Z, Mou T, Hu Z. Quantitative trait loci controlling $\mathrm{Cu}, \mathrm{Ca}, \mathrm{Zn}, \mathrm{Mn}$ and Fe content in rice grains. J Genet. 2008:87:305-10.

27. Zhong M, Wang L, Yuan J, Luo L, Xu C, He YQ. Identification of QTL affecting protein and amino acid contents in rice. Rice Sci. 2011;18:187-95.

28. Huang X, Zhao Y, Wei X, Li C, Wang A, Zhao Q, et al. Genome-wide association study of flowering time and grain yield traits in a worldwide collection of rice germplasm. Nat Genet. 2012;44:32-9. https://doi.org/10.1038/ng.1018.
29. Du J, Zeng D, Wang B, Qian Q, Zheng S, Ling HQ. Environmental effects on mineral accumulation in rice grains and identification of ecological specific QTLs. Environ Geochem Health. 2013;35:161-70. https://doi.org/10.1007/ s10653-012-9473-z PMID:22760687.

30. Gande NK, Kundur PJ, Soman R, Ambati R, Athanarayana R, Bekele BD, Shashidhar HE. Identification of putative candidate gene markers for grain zinc content using recombinant inbred lines (RIL) population of IRRI38 $x$ Jeerigesanna. African J Boitech. 2014;13(5):657-63.

31. Berhanu DB, Rakhia S, Naveen GK, Kundur PJ, Shashidhar HE. Estimationof genetic variability and correlation studies for grain zinc concentrationsand yield related traits in selected rice (Oryza Sativa L.) genotypes. Asian J Exp Biol Sci. 2013;4:391-7.

32. Nagesh P, Usharani G, Neeraja CN, Babu V, Reddy DT. Molecular mapping of high iron and zinc rich regions in rice (Oryza sativa L.) grains using microsatellite markers. Helix. 2013;2:231-7.

33. Kumar J, Jain S, Jain RK. Linkage mapping of grain iron and zinc content in F2 population derived from the cross between PAU201 and Palman579 in rice. Cereal Res Commun. 2014;42:389-400.

34. Lee G, Yun B, Kim K. Analysis of QTLs associated with the rice quality related gene by double haploid populations. Int J Genom. 2014;10:1155 https://doi. org/10.1155/2014/781832.

35. Zhang $\mathrm{M}$, et al. Mapping and validation of quantitative trait loci associated with concentrations of 16 elements in unmilled rice grain. Theor Appl Genet. 2014;127:137-65.

36. Swamy BPM, Kaladhar K, Anuradha K, Batchu KB, Longvah T, Sarla N. QTL analysis for grain iron and zinc concentration in two $\mathrm{O}$ nivara derived backcross populations. Rice Sci. 2018;25:1-11.

37. Flint-Garcia SA, Thornsberry JM, Buckler ES. Structure of linkage disequilibrium in plants. Annu Rev Plant Biol. 2003;54(1):357-74. https://doi. org/10.1146/annurev.arplant.54.031902.134907.

38. Roy JK, Bandopadhyay R, Rustgi S, Balyan HS, Gupta PK. Association analysis of agronomically important traits using SSR, SAMPL and AFLP markers in bread wheat. Curr Sci. 2006;90(5):683-9.

39. Pradhan SK, Barik SR, Sahoo A, Mohapatra S, Nayak DK, Mahender A, Meher J, Anandan A, Pandit E. Population structure, genetic diversity and molecular marker-trait association analysis for high temperature stress tolerance in rice. PLoS One. 2016;11:e0160027. https://doi.org/10.1371/ journal.pone.0160027.

40. Pandit E, Tasleem S, Barik SR, Mohanty DP, Nayak DK, Mohanty SP, Das S, Pradhan SK. Genome-wide association mapping reveals multiple QTLS governing tolerance response for seedling stage chilling stress in indica rice. Front Plant Sci. 2017;8:552. https://doi.org/10.3389/fpls.2017.00552.

41. Zhao K, Tung CW, Eizenga GC, Wright MH, Ali ML, Price AH. Genome-wide association mapping reveals a rich genetic architecture of complex traits in Oryza sativa. Nat Commun. 2011;2:467. https://doi.org/10.1038/ ncomms1467.

42. Huang Y, Sun C, Min J, Chen Y, Tong C, Bao J. Association mapping of quantitative trait loci for mineral element contents in whole grain rice (Oryza sativa L.). J Agric Food Chem. 2015;63:10885-92. https://doi.org/10. 1021/acs.jafc.5b04932

43. Agrama HA, Eizenga GC, Yan W. Association mapping of yield and its components in rice cultivars. Mol Breeding. 2007;19(4):341-56. https://doi. org/10.1007/s1 1032-006-9066-6.

44. Huang X, Wei X, Sang T, et al. Genome-wide association studies of 14 agronomic traits in rice landraces. Nat Genet. 2010;42:961-7.

45. Zhang T, Zhao X, Wang W, Pan Y, Huang L, Liu X, et al. Comparative transcriptome profiling of colding stress responsiveness in two contrasting rice genotypes. PLoS One. 2012;7:e43274.

46. Pan Y, Zhang H, Zhang D, Li J, Xiong H, Yu J, et al. Genetic analysis of cold tolerance at the germination and booting stages in rice by association mapping. PLoS One. 2015;10:e0120590. https://doi.org/10.1371/journal.pone. 0120590.

47. Zhao WG, Jong WC, Soon WK, Jeong HL, Kyung HM, Yong JP. Association analysis of physicochemical traits on eating quality in rice (Oryza sativa L.). Euphytica. 2013;191:9-21.

48. Kumar V, Singh A, Mithra ASV, Krishnamurthy SL, Parida SK, Jain S, et al. Genome-wide association mapping of salinity tolerance in rice (Oryza sativa). DNA Res. 2015;22:133-45. https://doi.org/10.1093/dnares/dsu046.

49. Muthukumar C, Subathra T, Aiswarya GV, Babu RC. Comparative genomewide association studies for plant production traits under drought in diverse rice (Oryza sativa L.) lines using SNP and SSR markers. Curr Sci. 2015. 109(1):139-47. 
50. Swamy BPM, Shamsudin NAA, Rahman SNA, Mauleon R, Ratnam W, Cruz MTS, Kumar A. Association mapping of yield and yield-related traits under reproductive stage drought stress in rice (Oryza sativa I.). Rice. 2017;10:21. https://doi.org/10.1186/s12284-017-0161-6.

51. Anandan A, Anumalla M, Pradhan SK, Ali J. Population structure, diversity and trait association analysis in rice (Oryza sativa L.) germplasm for Early Seedling Vigor (ESV) using trait linked SSR markers. PLoS ONE. 2016;11(3): e0152406. https://doi.org/10.1371/journal.pone.0152406.

52. Sellappan K, Datta K, Parkhi V, Datta SK. Rice caryopsis structure in relation to distribution of micronutrients (iron, zinc, b-carotene) of rice cultivars including transgenic indica rice. Plant Sci. 2009;177:557-62.

53. Jagadeesh BR, Krishnamurthy R, Surekha K, Yogesh GS. Studies on high accumulation of iron and zinc contents in some selected rice genotypes. Gobal J Bio Biotechnol. 2013;2(4):539-41.

54. Cakmak I, Torun A, Millet E, Feldman M, Fahima T, Korol AB. Triticum dicoccoides: an important genetic resource for increasing zinc and iron concentration in modern cultivated wheat. Soil Sci Plant Nutr. 2004;50: 1047-54.

55. Morgounov A, Gómez-Becerra HF, Abugalieva A, Dzhunusova M, Yessimbekova M, Muminjanov $\mathrm{H}$. Iron and zinc grain density in common wheat grown in Central Asia. Euphytica. 2007;155:193-203.

56. Johnson AAT, Kyriacou B, Callahan DL, Carruthers L, Stangoulis J. Constitutive overexpression of the OsNAS gene family reveals single-gene strategies for effective iron- and zinc-biofortification of rice endosperm. PLoS One. 2011;6(9):e24476.

57. Ishimaru Y, Bashir K, Nishizawa NK. Zn uptake and translocation in rice plants. Rice. 2011;4:21-7.

58. Fu Q, Zhang P, Tan L, Zhu Z, Ma D, Fu Y. Analysis of QTLs for yield-related traits in Yuanjiang common wild rice (Oryza rufipogon Griff.). J Geneics Genomics. 2010;37:147-57.

59. Shabbir MA, Anjum FM, Zahoor T, Nawaz H. Mineral and pasting characterization of Indica rice varieties with different milling fractions. Int J Agri Biol. 2008;10:556-60.

60. Nagesh V. Babu, RG. Usharani T, Reddy D. grain iron and zinc association studies in rice (Oryza sativa L.) F1 progenies. Arch Appl Sci Res. 2012;4(1): 696-702.

61. Anjum FM, Pasha I, Anwar Bugti M, Butt MS. Mineral composition of different Rice varieties and their milling fractions. Pak J Agri Sci. 2007:44(2): 332-6.

62. Babu RV. Importance and advantages of rice biofortification with iron and zinc. An open access journal published by ICRISAT. SAT eJ. 2013;11:1-6.

63. Roy SC, Sharma B. Assessment of genetic diversity in rice (Oryza sativa L.) germplasm based on agro-morphology traits and zinc-iron content for crop improvement. Physiol Moler Biol Plants. 2014;20(2):209-24.

64. Vishnu VN, Robin S, Sudhakar D, Rajeswari S, Raveendran M, Subramanian KS, Tannidi S, Balaji A. Genotypic variation for micronutrient content in traditional and improved Rice lines and its role in biofortification Programme. Indian J Sci Techn. 2014;7(9):1414-25.

65. N'Goran JAK, Laurent V, Risterucci AM, Lanaud C. The genetic structure of cocoa populations (Theobroma cacao L.) revealed by RFLP analysis. Euphytica. 2000;115:83-90

66. Suma MR. Identification of SSRs Based Candidate Gene Markers for Iron and Zinc Content in Rice (Oryza sativa L.) Using Bioinformatics Tools. Trends Biosciences. 2015:8:288-96.

67. Xu Q, Zheng T-Q, Hu X, Cheng L-R, Xu JL, Shi Y-M, et al. Examining Two Sets of Introgression Lines in Rice (Oryza sativa L.) Reveals Favorable Alleles that Improve Grain Zn and Fe Concentrations. PLoS ONE. 2015;10(7): e0131846. https://doi.org/10.1371/journal.pone.0131846.

68. Brait JF, Lobreaux S. Iron transport and storage in plants. Trends Plant Sci. 1997;2:187-93.

69. Pflieger S, Lefebvre V, Cause M. The candidate gene approach in plant genetics: a review. Mol Breed. 2001;7:275-91.

70. Kinoshita N, Kato M, Kopyasaki K, Kawashima T, Nishimura T, Hirayama Y, Takamure I, Sato T, Kato K. Identification of quantitative trait loci for rice grain quality and yield-related traits in two closely related Oryza sativa L. subsp. japonica cultivars grown near the northernmost limit for rice paddy cultivation. Breed Sci. 2017;67:191-206. https://doi.org/10.1270/jsbbs.16155.

71. Miura K, Ikeda M, Matsubara A, Song XJ, Ito M, Asano K, Matsuoka M, Kitano $\mathrm{H}$, Ashikari M. OsSPL14 promotes panicle branching and higher grain productivity in rice. Nat Genet. 2010;42:545-9.
72. Agarwal S, Venkata T, Kotla A, Mangrauthia SK, Neelamraju S. Expression patterns of QTL based and other candidate genes in Mdhukar x Swarna RILs with contrasting levels of iron and zinc in unpolished rice grains. Gene. 2014;546:430-6.

73. Cai H, Huang S, Che J, Yamaji N, Ma JF. The tonoplast-localized transporter OsHMA3 plays an important role in maintaining $\mathrm{Zn}$ homeostasis in rice. J Exp Bot. 2019;70(10):2717-25.

74. National Rice Research Institute, Cuttack, Odisha, India. Screening for micronutrient content. NRRI Annual report, 2013 (http://www.crri.nic.in).

75. National Rice Research Institute, Cuttack, Odisha, India. Screening for micronutrient content. NRRI Annual report, 2014 (http://www.crri.nic.in).

76. National Rice Research Institute, Cuttack, Odisha, India. Screening for micronutrient content. NRRI Annual report, 2015 (http://www.crri.nic.in).

77. Pradhan SK, Pandit E, Pawar S, Baksh SY, Mukherjee AK, Mohanty SP. Development of flash-flood tolerant and durable bacterial blight resistant versions of mega rice variety 'Swarna' through marker-assisted backcross breeding. Sci Rep. 2019;9:12810. https://doi.org/10.1038/s41598-019-49176-z.

78. Murray MG, Thompson WF. Rapid isolation of high molecular weight plant DNA. Nucleic Acids Res. 1980;8(19):4321-5 PMID: 7433111.

79. Liu K, Muse SV. PowerMarker: an integrated analysis environment for genetic marker analysis. Bioinformatics. 2005;21(9):2128-9. https://doi.org/10. 1093/bioinformatics/bti282.

80. Nei M. Genetic distance between populations. Am Nat. 1972;106:283-92.

81. Perrier $X$, Jacquemoud-Collet JP. DARwin software Available at http:// darwin.cirad.fr/darwin. 2006.

82. Pritchard JK, Stephens M, Donnelly P. Inference of population structure using multilocus genotype data. Genetics. 2000;155:945-59 PMID: 10835412.

83. Evanno G, Regnaut S, Goudet J. Detecting the number of clusters of individuals using the software STRUCTURE: a simulation study. Mol Ecol. 2005;14(8):2611-20. https://doi.org/10.1111/ j.1365-294X.2005.02553.x PMID: 15969739 .

84. Earl DA, Von HBM. Structure harvester: a website and program for visualizing structure output and implementing the Evanno method. Conserv Genet Resour. 2012;4(2):359-61. https://doi.org/10.1007/s12686-011-9548-7.

85. Peakall R, Smouse PE. GenAlEx 6.5, Genetic analysis in Excel. Population genetic software for teaching and research-an update. Bioinformatics. 2012; 28(19):2537-9. https://doi.org/10.1093/ bioinformatics/bts460 PMID: 22820204

86. Bradbury PJ, Zhang Z, Kroon DE, Casstevens TM, Ramdoss Y, Buckler ES TASSEL: software for association mapping of complex traits in diverse samples. Bioinformatics. 2007;23(19):2633-5 PMID: 17586829.

87. Yoshida S, Forno DA, Cock JAY, Gomez KA. Laboratory manual for plant physiological studies of rice. 3rd ed. Manila: International Rice Research Institute; 1976.

88. Pradhan SK, Pandit E, Nayak DK, Behera L, Mohapatra T. Genes, pathways and transcription factors involved in seedling stage chilling stress tolerance in indica rice through RNA-Seq analysis. BMC Plant Biol. 2019. https://doi. org/10.1186/s12870-019-1922-8.

89. Livak KJ, Schmittgen TD. Analysis of relative gene expression data using real-time quantitative PCR and the $2^{-\triangle \Delta C T}$ method. Methods. 2001;25:402-8.

\section{Publisher's Note}

Springer Nature remains neutral with regard to jurisdictional claims in published maps and institutional affiliations.

Ready to submit your research? Choose BMC and benefit from:

- fast, convenient online submission

- thorough peer review by experienced researchers in your field

- rapid publication on acceptance

- support for research data, including large and complex data types

- gold Open Access which fosters wider collaboration and increased citations

- maximum visibility for your research: over $100 \mathrm{M}$ website views per year

At $\mathrm{BMC}$, research is always in progress.

Learn more biomedcentral.com/submissions 